\title{
Numerical Simulation and Validation of the Aerodynamics of Static and Dynamic Spoilers*
}

\author{
Sven Geisbauer ${ }^{\dagger}$ \\ DLR - German Aerospace Center, 38108 Braunschweig, Germany
}

\begin{abstract}
Spoilers play a vital role in the flight control systems of modern transport aircraft. Due to their efficiency and fast deflection rates they are widely used to assist in roll control or for gust load alleviation purposes. Simulating the aerodynamic behavior of spoilers still is a challenging task as deflecting a spoiler always induces flow separation. The work presented in this paper therefore focused on extending the application range of DLR's in-house flow solver TAU by verifying and validating it for spoiler applications. In a first step, the work focused on steady and unsteady simulations of static and dynamic spoiler deflections in the low-speed regime. It was shown that the chosen numerical approach is well-suited to reproduce the surface pressure distribution for static spoiler deflections of up to $5^{\circ}$. Above that, the simulated transient aerodynamic response was found to be in good agreement with experimental data for a variety of deflection times and deflection angles.
\end{abstract}

\section{Nomenclature}

$$
\begin{array}{ll}
A R_{\mathrm{S}} & =\text { spoiler aspect ratio } \\
b & =\text { airfoil span, } \mathrm{m} \\
b_{\mathrm{S}} & =\text { spoiler span, } \mathrm{m} \\
C_{D} & =3 \mathrm{D} \text { drag coefficient } \\
C_{L} & =3 \mathrm{D} \text { lift coefficient } \\
c & =\text { airfoil chord length, } \mathrm{m} \\
c_{d} & =2 \mathrm{D} \text { drag coefficient } \\
c_{l} & =2 \mathrm{D} \text { lift coefficient } \\
c_{l, \text { adv }} & =2 \mathrm{D} \text { coefficient of maximum adverse lift } \\
c_{l, \text { final }} & =2 \mathrm{D} \text { lift coefficient at } t / t_{\mathrm{d}}=2 \\
c_{m} & =2 \mathrm{D} \text { pitching moment coefficient } \\
c_{p} & =\text { pressure coefficient }
\end{array}
$$

\footnotetext{
*Presented as Paper 2020-2776 at the virtual AIAA Aviation Forum 2020, 15-19 June 2020.

${ }^{\dagger}$ Research Engineer, Institute of Aerodynamics and Flow Technology, Lilienthalplatz 7, AIAA Senior Member.
} 


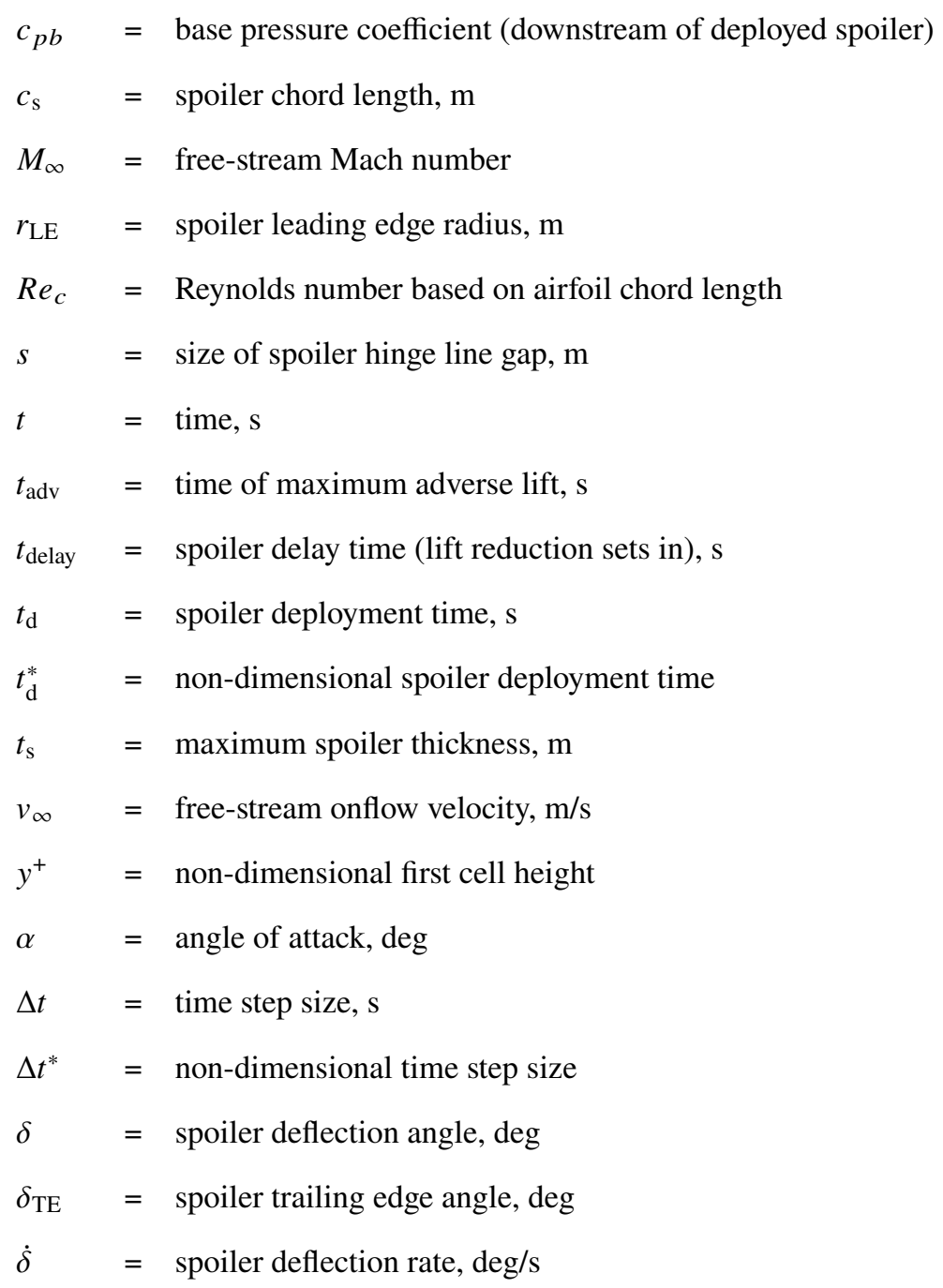

\section{Introduction}

mong all control surfaces of a modern transport aircraft spoilers are of particular interest from a modeling of tasks from more "traditional" applications such as speed brakes, supporting roll control or lift dumping to more advanced load alleviation functionalities [1, 2]. Their fast deflection rates and their ability to quickly redistribute the aerodynamic loads over a large portion of the wing has turned spoiler controls into the backbone of modern gust load alleviation (GLA) functionalities. On the other hand, their aerodynamic characteristics are challenging to model, as spoilers work by inducing a controlled flow separation over the wing. This typically leads to nonlinear aerodynamic characteristics and time delays, which prompted Mack et al. to state that “...their characteristics are the most difficult to predict of any of the control devices..." [3]. On top of that, when talking about dynamic spoiler deflections, the simulation tools needs to capture the transition from retracted state, i. e. the spoiler being flush with the surrounding 
wing surface, to deflected state. Nowadays, when simulating the aerodynamic behavior of moving spoilers flow solvers based on the Navier-Stokes equations in combination with overset grids are typically utilized, as for instance presented by Choi [4].

Until mid of the 1990's most work published on spoiler aerodynamics was done experimentally. Siddalingappa and Hancock [5] examined the nonlinearities and time lag effects of a two-dimensional spoiler, discovering that these effects were more pronounced in the presence of an already deflected flap or the more upstream the spoiler hinge line was located. They also found out that additional dynamic effects in terms of over- and undershoots can occur. This phenomenon, depicted in Fig. 1. was later called adverse lift and studied in more detail in the years to come.

\section{Lift coefficient}

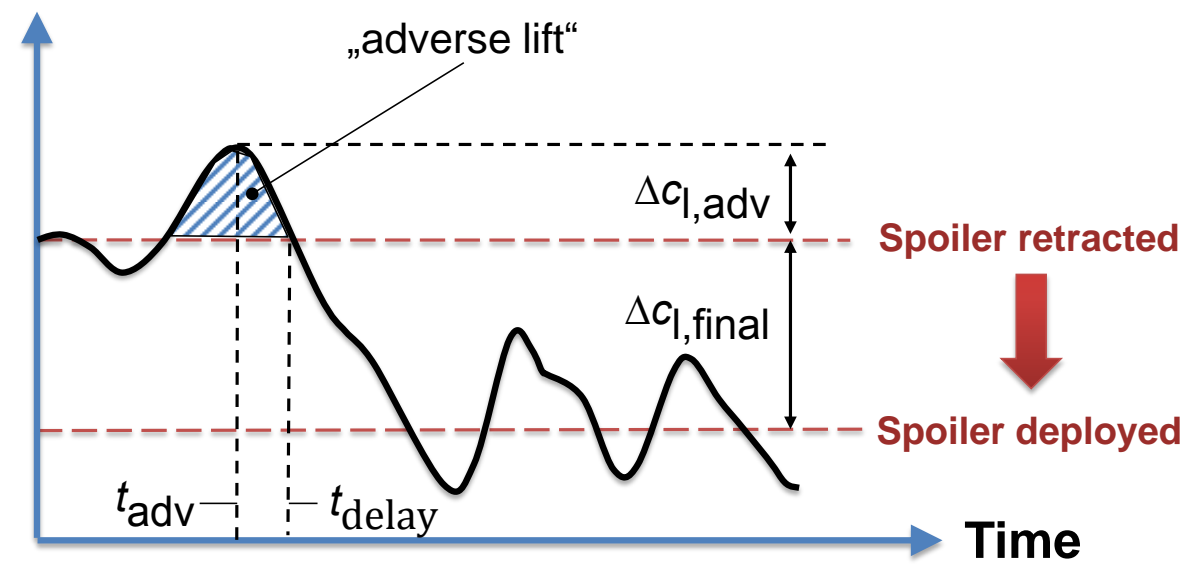

Fig. 1 Transient lift response due to a rapidly deploying spoiler.

Wentz et al. [6] investigated the impact of the spoiler geometry on its aerodynamic characteristics, finding out that the time delays could be reduced by introducing hinge line gaps, porosity or trailing edge modifications. Hancock [7] and Mabey [8] focused on the dynamic effects. While Hancock qualitatively described the physical causes leading to the aforementioned dynamic effects, Mabey introduced a criterion to determine whether or not they occur, Eq. (1), by setting the dimensional deflection time $t_{d}$ of the spoiler in relation to the convective time unit of the surrounding flow field. The latter is build with the chord length $c$ of the airfoil at the spanwise position of the spoiler and the undisturbed onflow velocity $v_{\infty}$.

$$
t_{\mathrm{d}}^{*}=t_{\mathrm{d}} \frac{v_{\infty}}{c}
$$

Now, according to Mabey, if the spoiler deflects within five convective time units, i. e.

$$
t_{\mathrm{d}}^{*}<5
$$

the spoiler is deflecting rapidly and the adverse dynamic effects shown in Fig. 11 will occur. 
Further experimental research focused on two-dimensional investigations of static [9-11], harmonically oscillating [12-14] or ramp-type [10, 12, 13, 15] spoiler deflections. Consigny et al. [12] found out that a rapid retraction of a spoiler, in contrast to its deflection, did not lead to any adverse effects. Kalligas [10] was among the first to also examine the drag response for rapid spoiler deflections, for which he noticed an even more sluggish behavior compared to the lift response. In contrast to Mabey's findings, he even observed adverse transient effects up to $t_{\mathrm{d}}^{*}=8$. The work of Yeung et al. [15] aimed at reducing the transient adverse effects of spoilers. They have demonstrated that introducing a gap at the spoiler hinge line is an effective means to achieve this. Their experimental data have been widely used by other researchers in the years to come for the validation of newly developed computational tools.

Experimental work involving finite, three-dimensional spoilers still is very limited. Jordan et al. [16] investigated the cause of unexpected dynamic loading conditions on the flap of a military aircraft, which had occurred during flight testing when the spoiler was deflected. They disclosed spoiler induced vortices interacting with the deployed flap as the physical cause for these conditions. By modifying the flap cove geometry, they managed to mitigate the problem. Scott et al. [17] presented a summary of aerodynamic data collected with the Benchmark Active Controls Technology (BACT) model, a generic wing configuration for research purposes with actuated spoiler and flap. Extensive data on this configuration have since been published, covering both static and harmonically oscillating spoiler and flap deflections up to the transonic flow regime [18]. However, no ramp-type spoiler deflections were investigated with that model.

Numerical activities dealing with moving spoilers have so far mostly been limited to two-dimensional cases, utilizing methods based on the Navier-Stokes equations with structured grids. Here again, the initial focus consisted in statically deflected spoilers [19], before harmonically oscillating spoilers were investigated [20]. Later on, the focus shifted towards rapid, ramp-type spoiler deflections, using structured overset grids [4, 21, 22]. In contrast to all of the aforementioned groups, $\mathrm{Xu}$ and Yeung [23] developed an alternative numerical approach, based on a discrete vortex model. Using this inviscid approach, they investigated both conventional and base-vented spoilers and were able to numerically reproduce the experimental findings of Yeung et al. [15] on how to reduce the adverse spoiler effects. Favre et al. [24] also used a different numerical approach by employing a method based on a combination of large-eddy simulations and immersed-boundary method with adaptive mesh refinement. They applied this approach to investigate rapid spoiler deflections for wind turbine applications and validated their tool with the experimental data by Yeung et al. [15].

Numerical activities found so far on three-dimensional spoilers exclusively address static spoiler deflections. With the exception of the work by Gand [25], who applied and compared a zonal detached-eddy simulation approach to RANS simulations for an aircraft with deployed spoiler, all others [26--28] have exclusively applied Navier-Stokes codes with structured grids. More recently, Tian et al. [29] applied a RANS method in steady and unsteady mode to investigate the potential of small spoiler deflections for buffet control on an airfoil and a wing, proving that small spoiler deflections could reduce the oscillation amplitudes at buffet onset. 
The aforementioned importance of spoilers in the flight control system of modern airliners, the challenges in simulating their aerodynamic behavior, the lack of available experimental data on ramp-type, finite spoiler deflections and the fact that little to none numerical work has so far been published on three-dimensional, rapidly deflecting spoilers all gave reason for conducting a comprehensive research campaign on spoiler aerodynamics, both experimentally and numerically. The final objective of this work consisted in extending the application range of the DLR TAU code by verifying and validating it for spoiler applications. A summary of these efforts is presented in the following.

\section{Reference Experiment}

The model used in this work is the so-called DLR-F15DS (dynamic spoiler) model, an assembly of a 2D wall-to-wall model in clean configuration with a constant chord of $c=600 \mathrm{~mm}$ and a span of $b=2800 \mathrm{~mm}$ and an embedded and actuated, finite spoiler panel. The spoiler was integrated symmetrically around the mid-span position of the F15DS model and is hinged at $x / c=0.745$. It features a chord length of $c_{s} / c=0.15$ and a span of $b_{s} / b=0.214$, leading to a spoiler aspect ratio of $A R_{s}=6.7$. Hence, due to the finite spanwise extension of the spoiler, it will induce a $3 \mathrm{D}$ flow field when deployed. Figure 2 shows a sectional view of the F15DS airfoil with deployed spoiler.

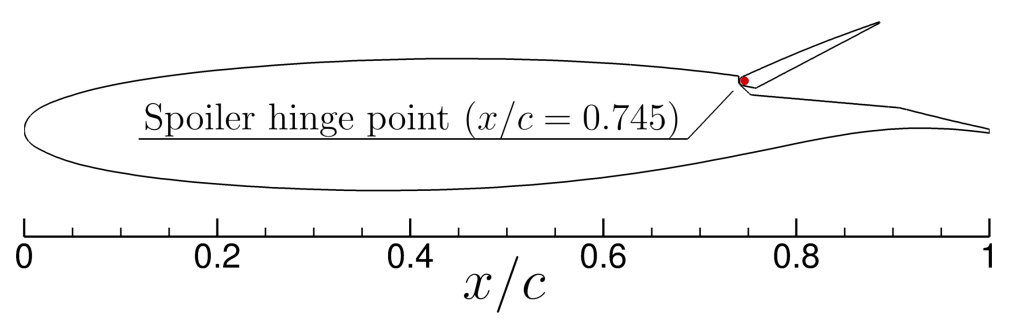

Fig. 2 Sectional view at the DLR-F15DS model with deflected spoiler.

The F15DS model was equipped with a total of 150 static pressure taps, distributed over three sections. The center section in the symmetry plane is the main section of interest and features the densest distribution, with four pressure taps on the front face of the spoiler. Two additional sections with a less dense distribution were located symmetrically to the center section, in close proximity to the upper and lower wind tunnel walls to check for symmetric onflow conditions. In addition, 30 unsteady pressure transducers were distributed along the center section to collect transient pressure data during spoiler deployment. The spoiler was actuated by an electrically driven linear actuator unit which, due to space constraints, was mounted below the floor of the test section, not in the model itself. Using a push rod and a lever kinematics within the model, the linear motion of the actuator was evenly transformed into a rotational motion of the spoiler, enabling arbitrary deflection angles up to $85^{\circ}$. To account for mechanical play in the kinematics the actual position of the spoiler was additionally tracked with an external 3D video tracking system which relied on the stereo pattern recognition (SPR) technique. This system worked at a frame rate of 1,000 fps. The signals of the unsteady pressure transducers were sampled at $1000 \mathrm{~Hz}$ and low-pass filtered with a cutoff frequency of $391 \mathrm{~Hz}$ before 
analog-digital conversion. Transition was fixed at $x / c=0$ using an adhesive tape with a height of $0.2 \mathrm{~mm}$ and a width of $3.0 \mathrm{~mm}$. As the model was not equipped with a wind tunnel balance, the validation of the DLR TAU code relies on the measurements of steady and unsteady surface pressure data and the integrated sectional force and moment coefficients. An overview on the DLR-F15DS geometry in assembled state is shown in Figs. 3 and 4

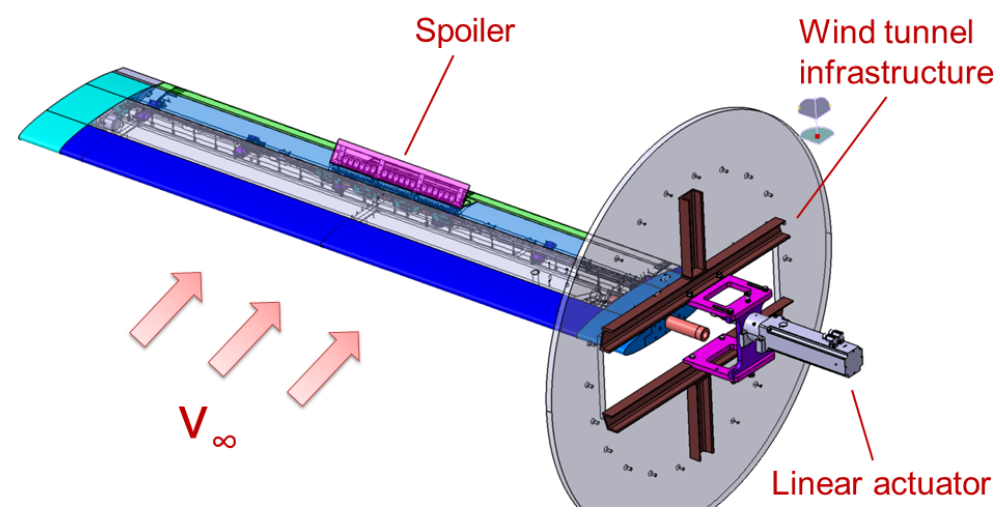

Fig. 3 CAD model of the DLR-F15DS wind tunnel model with deflected spoiler.

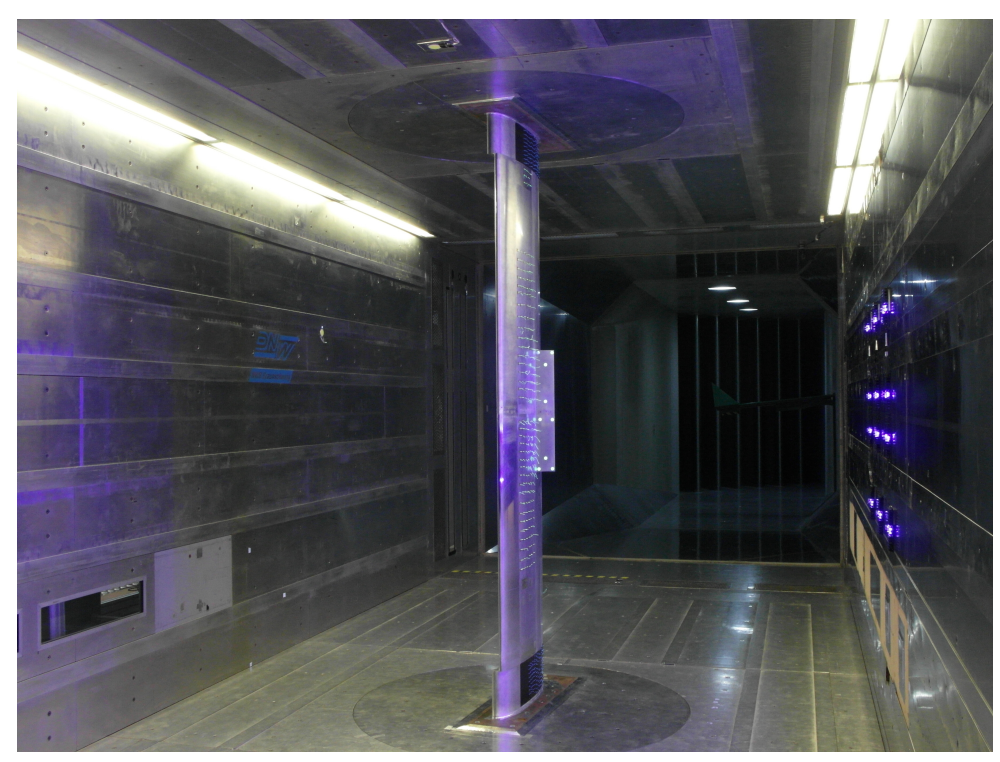

Fig. 4 View in downstream direction on the vertically mounted model in the DNW-NWB tunnel.

\section{A. Wind Tunnel Test Conditions}

The tests were conducted in the low-speed wind tunnel Braunschweig of the German-Dutch Wind Tunnels (DNWNWB). As shown in Fig. 4 the model was mounted vertically in the closed test section. The runs used for the validation were conducted at $M_{\infty}=0.2$, which corresponds to a Reynolds number based on the chord length of the airfoil of $R e_{c}=2.6 \mathrm{~m}$. Before investigating different spoiler deflections the clean configuration was examined from $\alpha=-5^{\circ}$ to post stall conditions and back again. Static spoiler deflections were investigated from $\delta=2.5^{\circ}$ up to $85^{\circ}$. Dynamic, 
ramp-type deflections were conducted from retracted state to $\delta=10^{\circ}, 30^{\circ}$ and $50^{\circ}$. During these dynamic deflections the non-dimensional deployment times according to Eq. (1) were gradually reduced from $t_{\mathrm{d}}^{*}=57$ to $t_{\mathrm{d}}^{*}=7$ to trigger the adverse lift phenomenon.

Table 1 Uncertainty of the pressure sensors in determining $c_{p}$ at $M_{\infty}=0.2$.

\begin{tabular}{lc}
\hline \hline Type & $\Delta c_{p}$ \\
\hline Static pressure ports / PSI modules & $0.01-0.04$ \\
Unsteady pressure transducers & $0.04-0.11$ \\
\hline \hline
\end{tabular}

\section{B. Accuracy Considerations}

The accuracy of the pressure sensors in terms of $c_{p}$ at $M_{\infty}=0.2$ is summarized in Table 1 . The given range of accuracy is due to different types of PSI modules and unsteady transducers used. After calibrating the actuator a maximum deviation between target and actual spoiler angle was found to be $\Delta \delta_{\text {Actuator }}= \pm 0.4^{\circ}$. This difference is due to mechanical play in the kinematics. For validation purposes, however, this information is irrelevant as the validation would be based on comparing the aerodynamic characteristics at a given measured spoiler angle. The latter was measured by the SPR video tracking system, with an accuracy, i. e. a maximum difference between measured and actual angle, in the range of $\Delta \delta_{\mathrm{SPR}}= \pm 0.5^{\circ}$. Wind tunnel blockage effects were considered to be small. Hence, corrections of any blockage effects were not applied and uncorrected wind tunnel data were used to validate the TAU code. Further details on the experimental setup, the instrumentation and the measured data were published by the author in [30].

\section{Numerical Approach}

The flow simulations were conducted with the DLR TAU code, a hybrid, unstructured RANS solver for threedimensional, time-accurate and compressible flows, being under continuous development by DLR [31]. TAU was used in cell-vertex mode, the spatial discretization was carried out with a central scheme with artificial matrix dissipation. A semi-implicit approach was chosen for discretizing the time derivative, using a backward difference formulation in conjunction with a Lower-Upper Symmetric Gauss-Seidel scheme.

Three different turbulence models were used. The first was a modified, negative version of the model by Spalart and Allmaras (SA-neg) [32], which nowadays has become the standard one-equation model in TAU. Secondly, the two-equation shear stress transport model (SST) by Menter [33] was used, as it is generally known for its advantages in predicting flows with adverse pressure gradients. It was therefore used to investigate its suitability in predicting the pressure induced separation bubble immediately upstream of the spoiler hinge line at moderate and large spoiler deflections. The third was the differential SSG/LRR-g Reynolds stress model (RSM) according to Togiti and Eisfeld [34], which is a modified version of the model developed by Cecora et al. [35]. All simulations were performed fully 
turbulent.

The unsteady computations were carried out using a dual-time stepping scheme, based on a pre-converged steady flow solution. The time step size was chosen such that a mean change in spoiler deployment angle of $\Delta \delta=0.25^{\circ}$ was achieved per time step. For instance, the deployment of a spoiler from $\delta=0^{\circ}$ to $30^{\circ}$ in $60 \mathrm{~ms}$ would be computed with a time step size of $\Delta t=0.5 \mathrm{~ms}$, leading to a non-dimensional time step of $\Delta t^{*}=0.06$ according to Eq. (1). A further reduction, for instance an angular resolution of $0.125^{\circ}$ per time step, did not yield any benefits in predicting the transient force and moment coefficients, as will be shown later in sectionV.A

To save computational time a Cauchy convergence criterion was applied. It monitors the convergence history of lift and drag coefficients over the number of inner iterations. As soon as the remaining fluctuations within the last 250 inner iterations were below a certain threshold compared to the respective mean values within that window, i. e. $\Delta C_{L}<10^{-4}$ and $\Delta C_{D}<5 \cdot 10^{-5}$, the current time step was considered to be converged and the physical time was advanced by $\Delta t$.

The transient motion of the spoiler was controlled by the motion law given by Eqs. (3) and (4), which originates from the work of Consigny et al. [12]. It provides a smooth variation of the spoiler angle and its angular velocity. In addition, it approximates the acceleration and deceleration phase of real actuators very well. For that reason it has become a standard when simulating deflecting control surfaces. The deflection time $t_{\mathrm{d}}$ and the target deflection angle $\delta_{\text {target }}$ are the only two control inputs when dealing with deflections from fully retracted state.

$$
\begin{array}{ll}
\delta(t)=\delta_{\text {target }} / 2\left[1-\cos \left(\pi t / t_{\mathrm{d}}\right)\right] & \text { for } 0<t<t_{\mathrm{d}} \\
\delta(t)=\delta_{\text {target }} & \text { for } t \geq t_{\mathrm{d}}
\end{array}
$$

The spoiler deflection rate was automatically computed as the time derivative of Eq. (3) and both the instantaneous spoiler angle $\delta(t)$ and the deflection rate $\dot{\delta}(t)$ were provided as input for TAU.

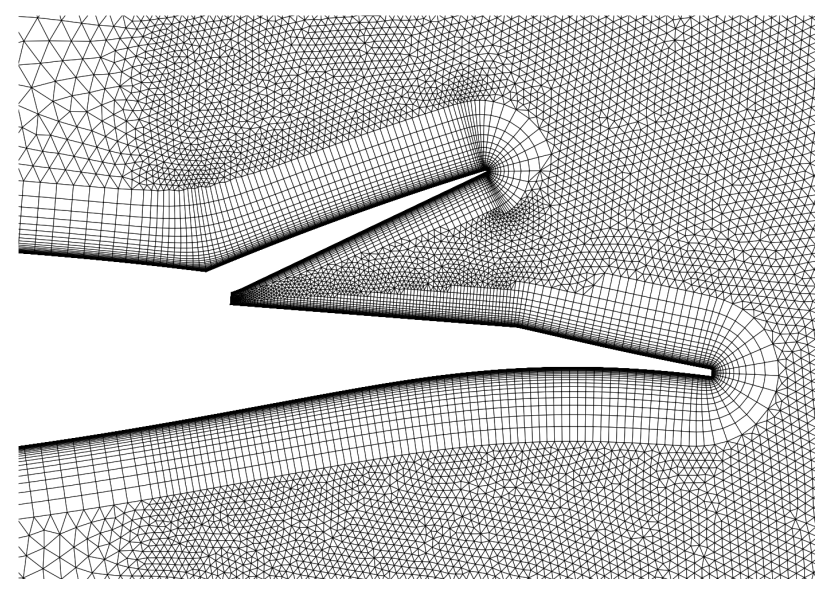

Fig. 5 One-block grid for static spoiler-out cases without spoiler hinge line gap.

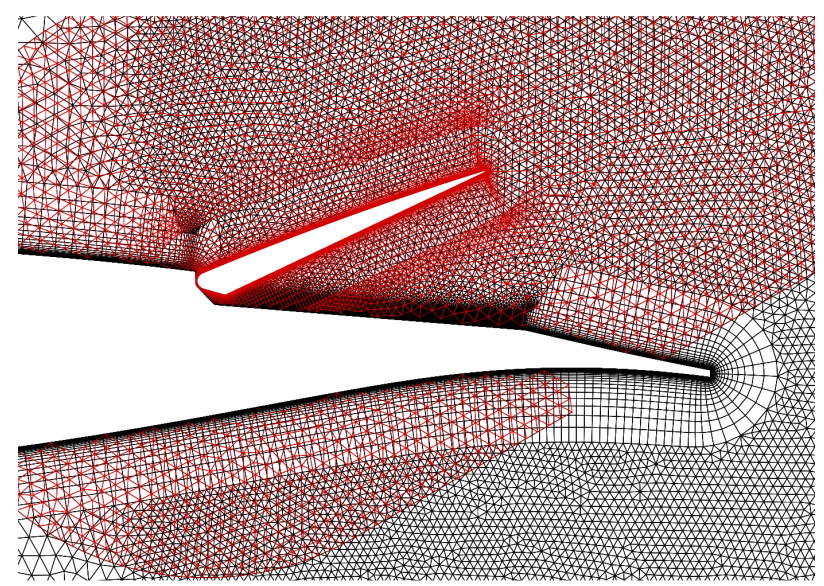

Fig. 6 Two-block overset grid for static and dynamic spoiler-out cases with hinge line gap after hole-cutting. 


\section{A. Grid Generation}

Two different meshing strategies were pursued: for static spoiler-out cases conventional unstructured, hybrid 3D one-block grids were generated in a first step, to simplify the grid generation process and to gather first experience in dealing with flows past deflected spoilers. As depicted in Fig.5, the spoiler is trimmed with the upper wing surface, i. e. there is no spoiler hinge line gap. The prismatic near-wall mesh was generated in such a way to yield a non-dimensional first wall spacing of $y^{+} \approx 1$ at $M_{\infty}=0.2$ and $R e_{c}=2.6 \mathrm{~m}$. The tetrahedral volume mesh was refined from $x / c=0.72$ to 4.0 to better capture the spoiler wake, with an element size linearly increasing in downstream direction, from $0.003 c$ to $0.017 c$.

For dynamic spoiler deflections a different approach had to be applied, still relying on unstructured grids. The grid deformation or the overset grid techniques are the general methods of choice when moving control surfaces are to be simulated. Due to the limitations of the grid deformation technique for deflections beyond around $30^{\circ}$ to $40^{\circ}$ and, more importantly, due to the fact that a full retraction of the spoiler into the wing is hardly possible, the overset grid technique was chosen here. In its current TAU implementation, this technique requires that there must exist a small gap between moving bodies at all times. The size of this gap, which is illustrated among other relevant geometric parameters in Fig.7, amounts to $s / c=0.083 \%$.

The final overset grid is composed of separately meshed component grids of wing and spoiler, as shown above in Fig. 6 Nearly the same settings as in the one-block grids were used for generating the overset meshes, with the exception that additional sources were required to refine the gap regions. Both meshes heavily relied on structured elements for the discretization of the surfaces.

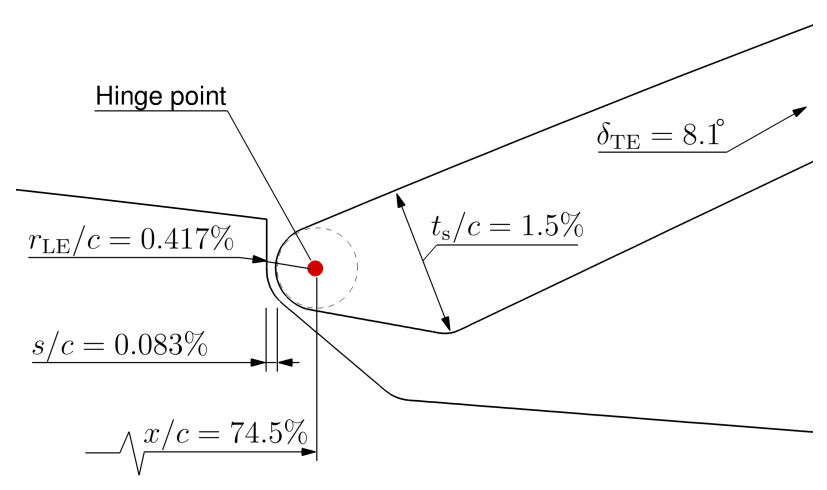

Fig. 7 View on the hinge line region of the overset grid.

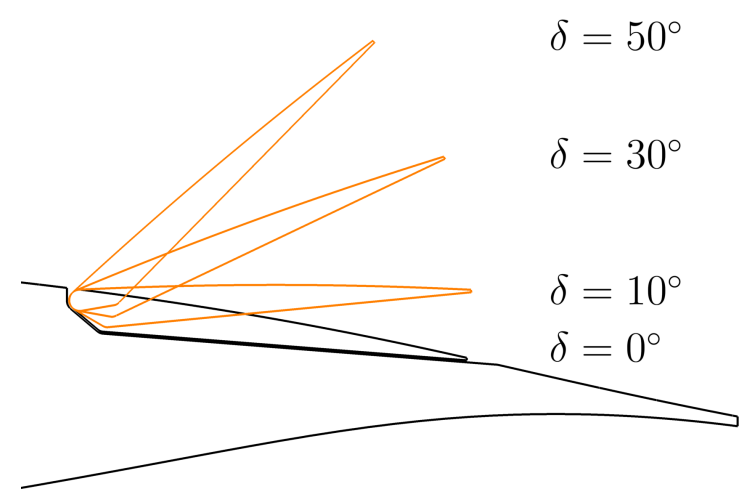

Fig. 8 Spoiler deflection angles investigated in this work.

The flow quantities are then interpolated between the meshes. The TAU implementation requires an overlap of at least two cells, which should ideally be of similar size, to allow for a second-order spatial discretization in the overlap region. This requirement must also be fulfilled in the gap region, leading to tiny cell sizes. Therefore, the overall number of points has nearly doubled compared to the size of the one-block grid, while the spatial discretization in the main area 
of interest, i.e. upstream, around and downstream of the spoiler, nearly remains constant in both cases. The final overset grid consists of $20 \mathrm{~m}$ nodes, whereas the initial one-block grid, in the following called medium grid, has around $10.5 \mathrm{~m}$ nodes. Based on the medium grid, both a coarsened and a refined grid were generated with a halved/doubled number of grid nodes to investigate the influence of the spatial discretization.

When using overset grids the inevitable situation occurs that parts of a component grid overlap with the geometry of another component. For example, the red spoiler component grid shown in Fig.6 6initially protrudes the wing geometry, resulting in grid points lying within a closed geometry. The hole-cutting process avoids this situation by identifying grid points which lie within a geometry and by excluding them from the computation. In this work the fully automatic hole-cutting algorithm developed by Spiering [36] was applied. Figure 6] shows the situation after hole-cutting.

\section{Static Spoiler Deflections}

The verification and validation results are presented in the following, first for static spoiler deflections, then for dynamic deflections. The investigated variations in spoiler deployment angle are illustrated in Fig. 8. Steady TAU computations were conducted for all cases with static spoiler deflections, despite the challenging flow field induced by them. This choice was made deliberately for two reasons: first of all, other researchers have already revealed promising results in applying steady RANS methods to configurations with spoilers deployed up to $45^{\circ}$ [28, 37]. Secondly, and encouraged by their results, the objective in this work consisted in benchmarking TAU and gain experience on the accuracy and the limitations of the steady approach for this kind of applications. All simulations were conducted at $M_{\infty}=0.2$ and $R e_{c}=2.6 \mathrm{~m}$.

\section{A. Grid Resolution Influence}

The influence of the grid resolution on the surface pressure distribution was examined for the case with the highest spoiler deflection at $\delta=50^{\circ}$. A comparison between TAU results, using the SA-neg turbulence model, and experimental data is shown in Fig. 9 with the black triangles indicating the data from the PSI modules (static pressure only) and the light blue circles indicating the mean static pressure as measured by the unsteady pressure transducers.

In general, the numerical results are in good agreement with the experiments on all three grids. Differences exist near the spoiler hinge line and in the base pressure region behind the deployed spoiler. In the latter region, at $x / c>0.8$, TAU consistently predicts lower pressure levels. This behavior was found to be independent of the spoiler deflection angle, the angle of attack or the turbulence model used in the simulation. As will be shown in section IV.C the accuracy in that region could only be improved when simulating time-accurately.

The differences between the different grid levels become more evident when the angle of attack is increased to $\alpha=10^{\circ}$, as shown in Fig. 9(b) While the base pressure is completely unaffected by the grid resolution, a stronger influence can be observed near the spoiler hinge line, between $0.6 \leq x / c \leq 0.8$. In fact, this region was found to be the 


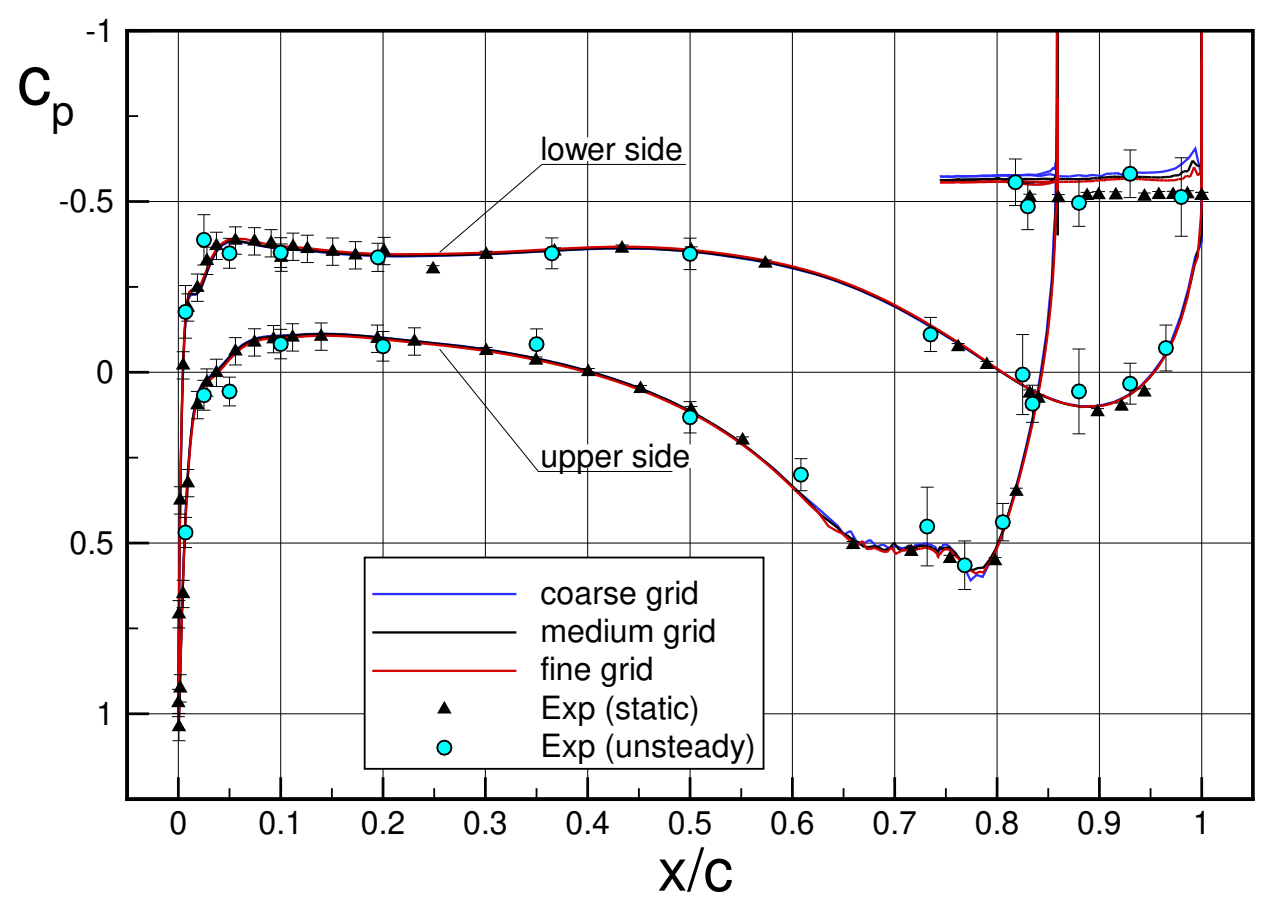

(a) $\alpha=0^{\circ}$.

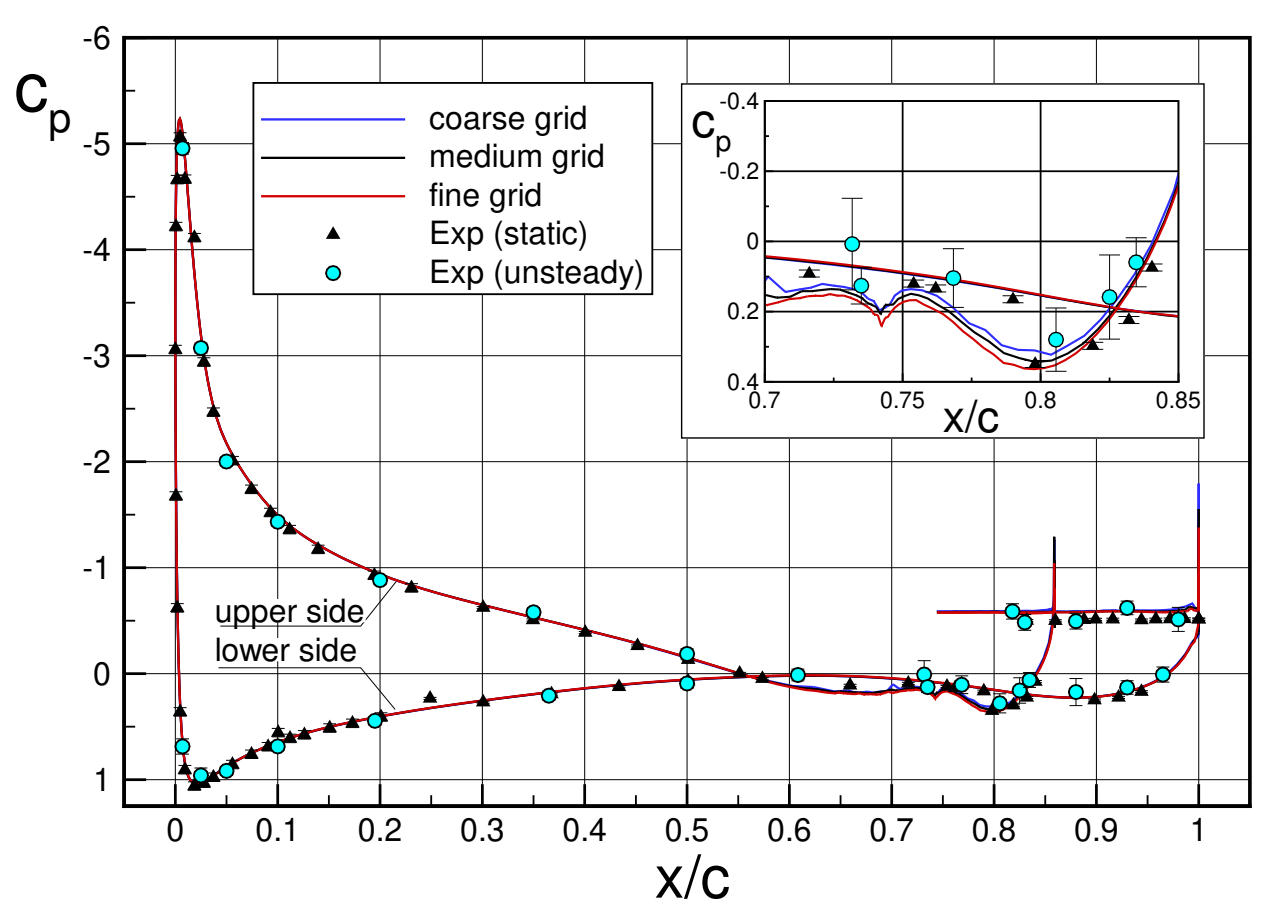

(b) $\alpha=10^{\circ}$.

Fig. 9 Grid resolution influence at $\delta=50^{\circ}$, using the SA-neg model.

only one on the entire configuration where distinct mesh influences occurred. As the grid influence on the pressure distribution was considered small, which especially holds for the medium and the fine grid, it was decided to use the 
medium one-block grid for the subsequent validation efforts of TAU.

\section{B. Turbulence Model Influence}

The influence of the three turbulence models was investigated at $\delta=10^{\circ}, 30^{\circ}$ and $50^{\circ}$. In general, it can be summarized that the pressure distribution was predicted very well in qualitative terms by all turbulence models, independent of angle of attack or spoiler deflection angle. As an example, Fig. 10 shows the pressure distributions at two different angles of attack at $\delta=30^{\circ}$. Nonetheless, quantitative differences occur in predicting the pressure at the hinge line and behind the deployed spoiler, with maximum differences in those regions of $\Delta c_{p}=0.07$ between the SA (lowest pressure) and the RSM results (highest pressure). In that case the RSM result is in slightly better agreement with experimental data. Deviations can also be observed near the spoiler hinge line, between $0.68 \leq x / c \leq 0.8$. They are due to a differently sized separation bubble in that region. The RSM predicts a slightly larger separation bubble in that region, whereas the SA-neg produces the smallest. Hence, the SA-neg result is either on par with the others or even matches experimental data slightly better in that region. The base pressure at the back side of the deployed spoiler is predicted similarly by all three turbulence models, however, with pressure levels being generally lower by $\Delta c_{p}=0.08-0.10$.

The higher the spoiler deflection the more pronounced are the differences in the two aforementioned regions. Figure 11 shows the corresponding diagram at $\delta=50^{\circ}$, focusing on the rear part of the pressure distribution. The SA-neg and SST models produce a more oscillatory behavior at the spoiler hinge line, whereas the RSM shows a smoother transition onto the reattachment point on the spoiler. Compared to experimental data, all models produce satisfying results. It must be noted, however, that the distribution of the static pressure ports was not dense enough to finally judge on which model performs best in that region. Behind the spoiler, all models still overestimate the intensity of the low pressure region. However, as shown in Fig. 11, the SA-neg is closest to experimental data.

The influence of the turbulence models on the integrated sectional force and moment coefficients is summarized in Fig. 12 for $\alpha=0^{\circ}$. Over the complete range of investigated spoiler deflections all turbulence models underestimate the sectional lift, with the SA-neg producing the most accurate results with a maximum deviation of just above 5\% to experimental data at $\delta=30^{\circ}$.

In contrast, the RSM underpredicts the lift by more than $20 \%$ at $\delta=10^{\circ}$ and overpredicts it by the same percentage at $\delta=50^{\circ}$. While the reason for the former is not known yet, the latter is most probably due to a rather poor convergence behavior of the RSM simulations. The SST yields similarly large differences at deflections of $10^{\circ}$ and $30^{\circ}$, although it still converges very well in both cases.

A comparison of the absolute drag coefficients needs to be taken with caution as the experimental data only accounts for the pressure part of the drag while neglecting the viscous contribution. For that reason, all CFD results show higher drag coefficients, with the SST performing slightly better than the SA-neg at $\delta=30^{\circ}$. However, the SA is closest to 


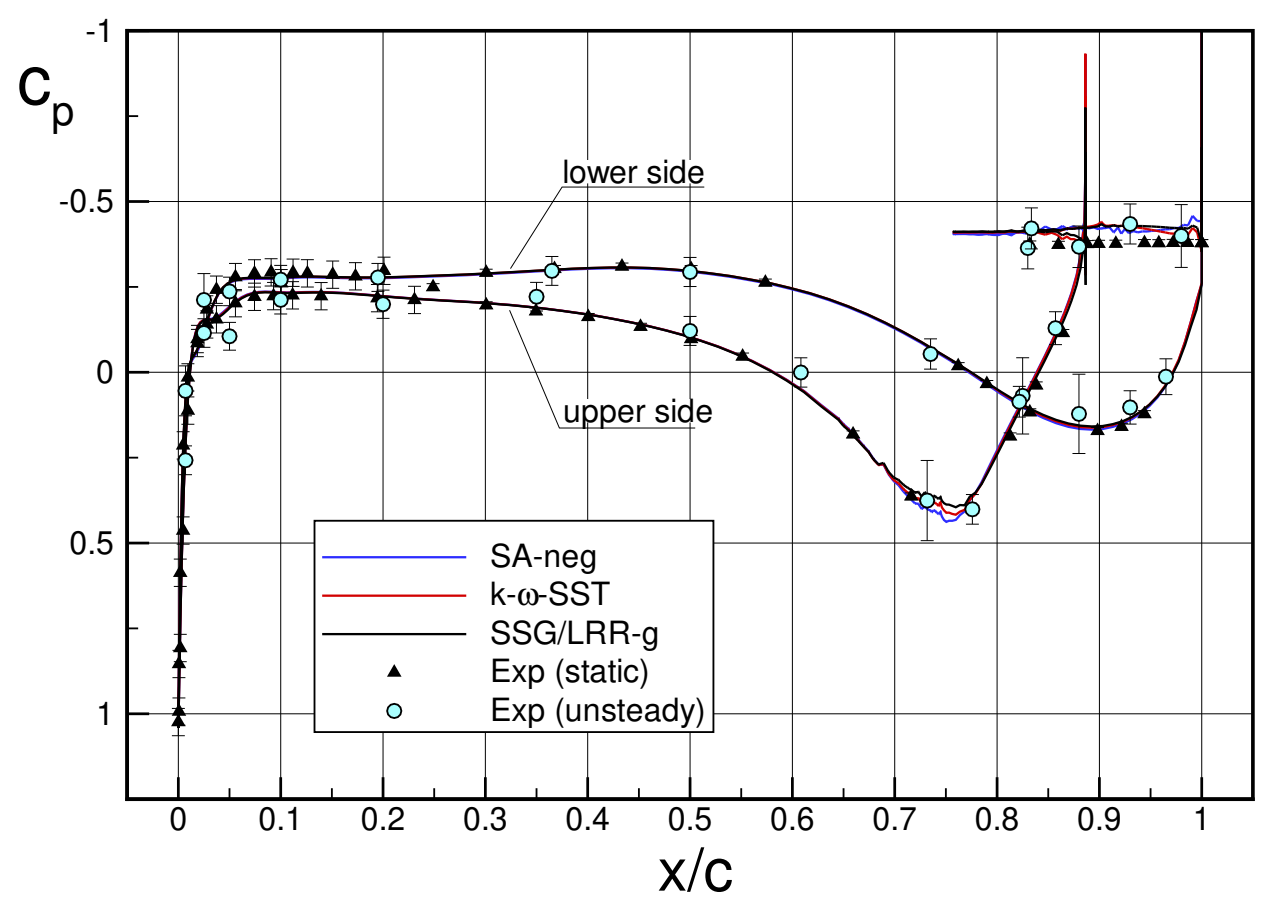

(a) $\alpha=0^{\circ}$.

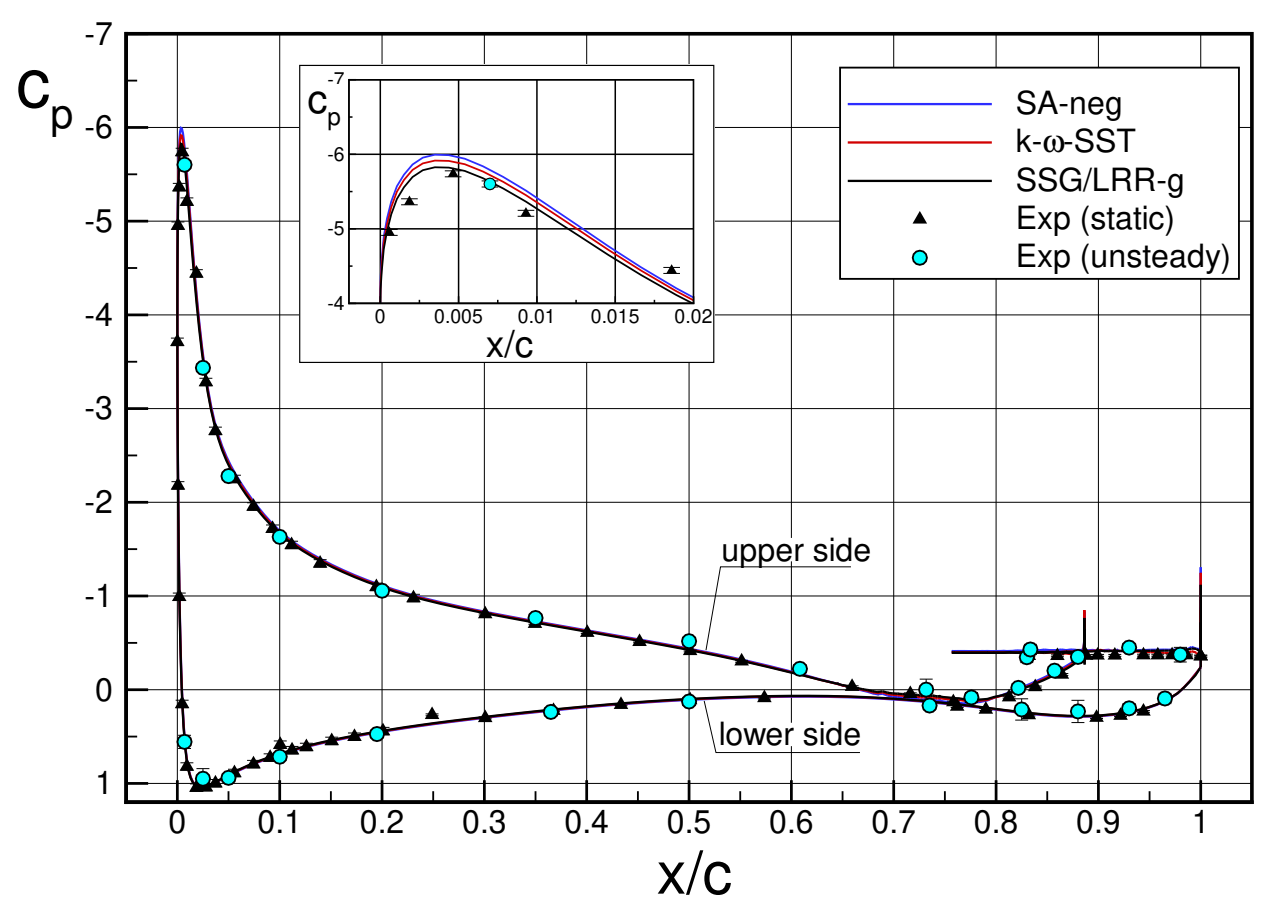

(b) $\alpha=10^{\circ}$.

Fig. 10 Turbulence model influence on pressure distribution at $\delta=30^{\circ}$.

experimental data again at $\delta=50^{\circ}$.

As shown the SA-neg shows the most consistent behavior at this angle of attack and, in many cases, was closest to 


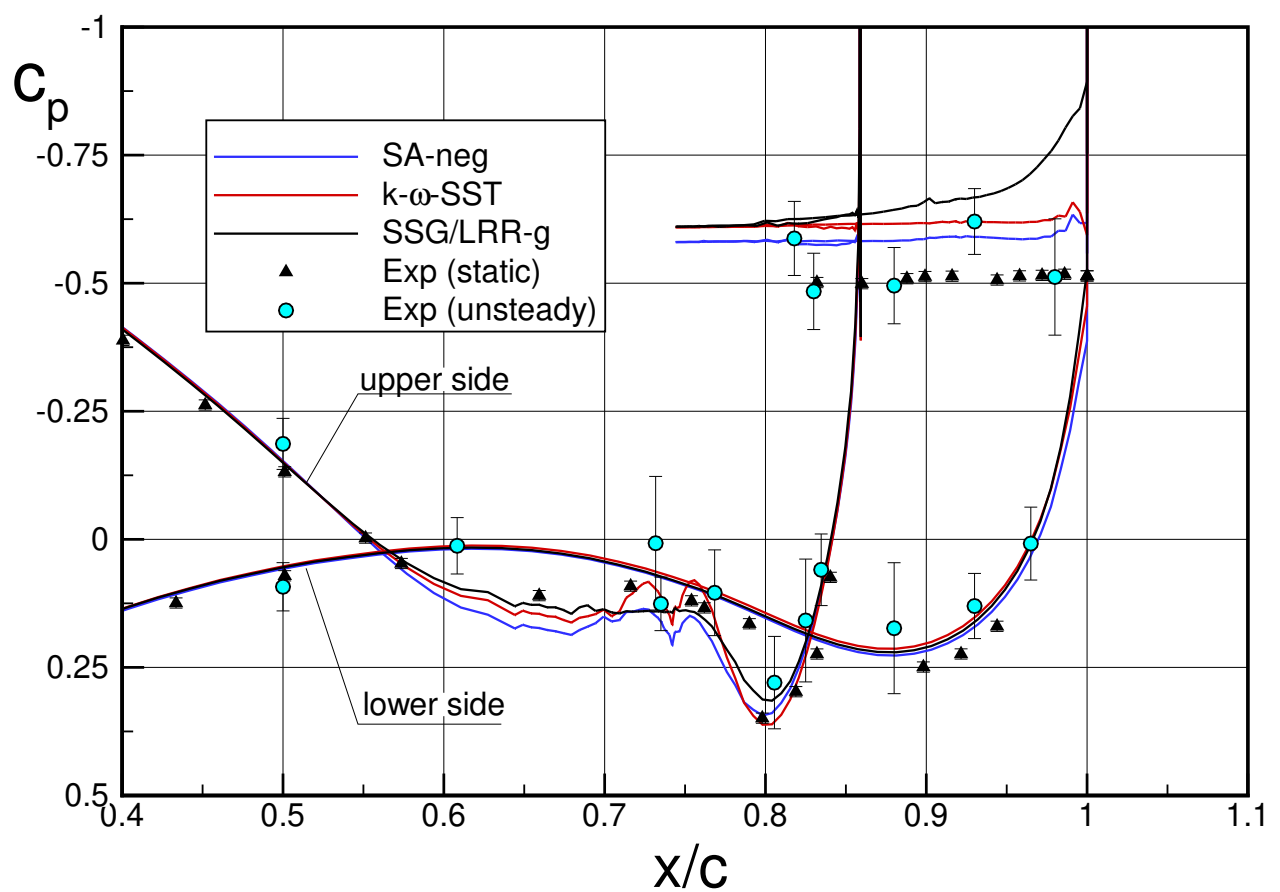

Fig. 11 Turbulence model influence on pressure distribution at $\delta=50^{\circ}$ and $\alpha=10^{\circ}$.

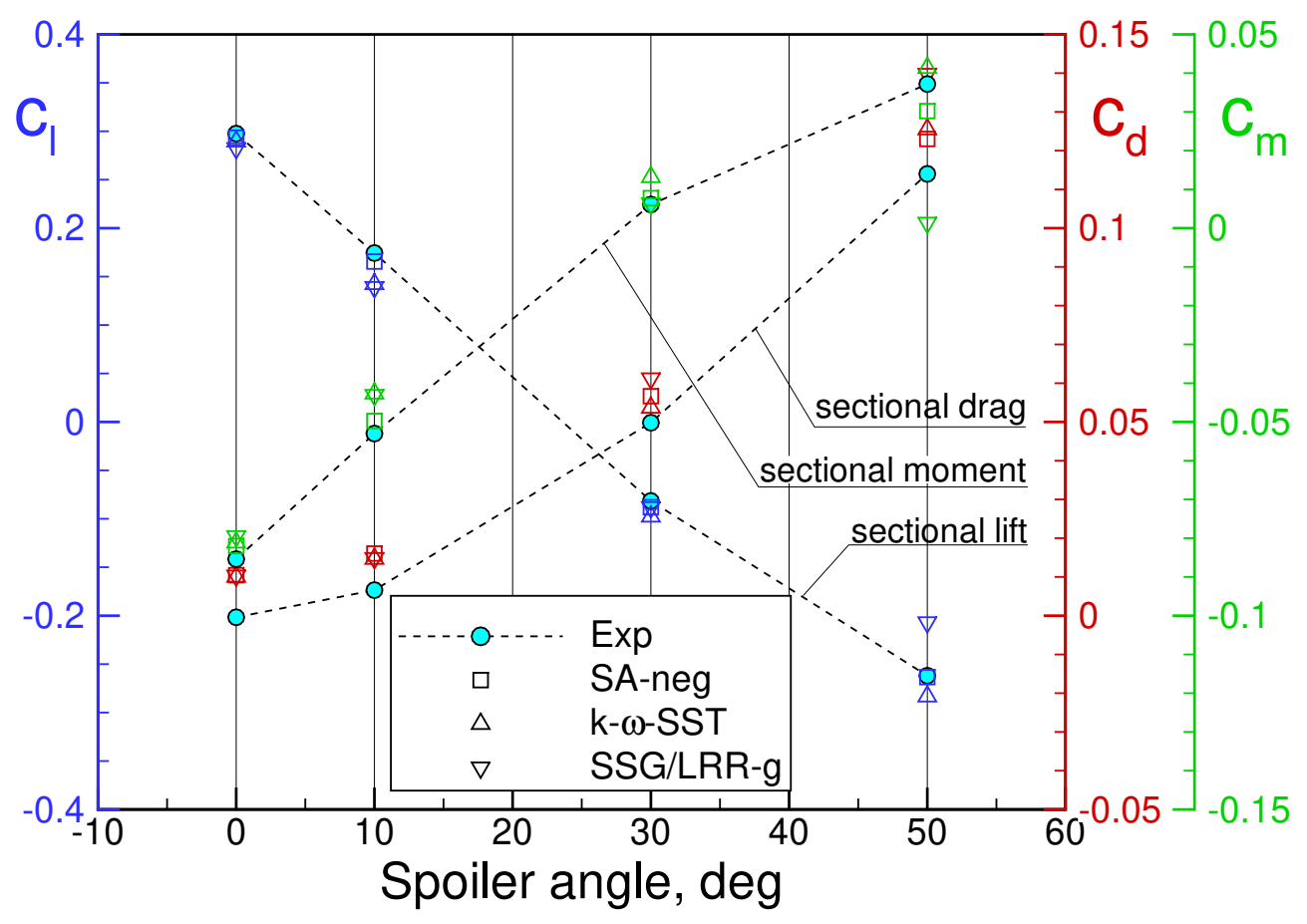

Fig. 12 Summary of turbulence model influence on sectional force and moment coefficients at $\alpha=0^{\circ}$. 
experimental data. Although not explicitly shown here preliminary results at $\alpha=10^{\circ}$ reveal that the situation changes at higher angle of attack, with the RSM now showing the most consistent and most accurate performance.

To summarize, all turbulence models were well-suited to predict the surface pressure distribution on this configuration with deployed spoiler in qualitative terms. No model produced results being completely off and if the objective of a CFD study consisted in investigating incremental changes only, one would probably achieve acceptable and consistent results with each of them. When quantitative accuracy is the key metric, the RSM was found to be superior for high angles of attack, especially because of its more accurate prediction of the suction peak at the leading edge. At low angles of attack, the SA-neg was mostly on par or even more accurate than the others, especially in predicting the correct base pressure at higher spoiler deflections. Due to its consistent performance, its robustness and its computational efficiency (2,3x faster than RSM) it was decided to rely on the SA-neg model for the remainder of this work.

\section{Influence of Time-Accurate Simulations}

As soon as the spoiler is deflected the flow past its tip will separate. However, depending on the spoiler geometry and the onflow conditions, the flow might reattach before reaching the trailing edge. As published in [30], it was observed in the reference experiment that the flow reattaches for $\alpha \leq 10^{\circ}$ and $\delta<5^{\circ}$. The smallest spoiler deflection angle investigated in the validation, however, was already at $\delta=10^{\circ}$. It can therefore be assumed that (a) the flow past the spoiler is fully separated in all cases considered within this validation and (b) that there is an interaction of the flow past the upper and lower sides of the airfoil, leading to a vortex-dominated flow topology in the wake of the spoiler as shown in Fig. 13 .

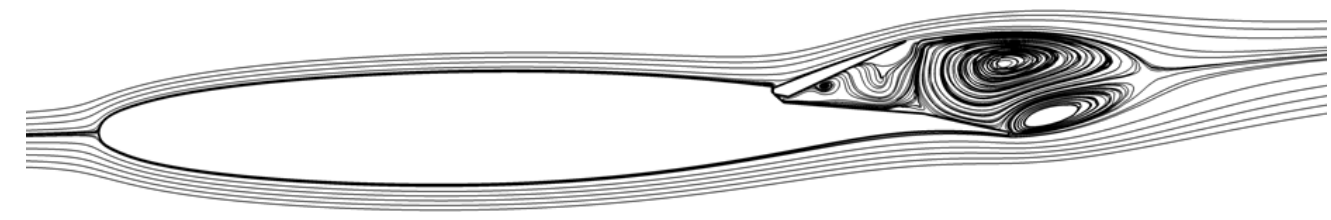

Fig. 13 Typical mean streamline pattern around a spoiler deflected to $\delta=30^{\circ}$ and $\alpha=0^{\circ}$.

Predicting this flow topology certainly exceeds the usual application range of steady RANS methods as the main criterion to apply these methods, i. e. a largely attached flow field, is no longer fulfilled. Despite the fact that the so-far used steady simulation approach was able to compute pressure distributions which to a large extent were in good agreement with experimental data, the simulations consistently predicted are more negative base pressure coefficient, i. e. a stronger suction force downstream of the spoiler. As shown in the previous sections, neither a higher spatial resolution nor a more advanced turbulence model improved the accuracy in that region. For that reason, and as a preparatory step for the subsequent dynamic spoiler-out cases, it was investigated if time-accurate RANS (uRANS) simulations for static spoiler-out cases would improve the accuracy of the simulation, especially in the base pressure region.

Using steady restart solutions at $\alpha=0^{\circ}$ and $10^{\circ}$ uRANS simulations were carried out for spoiler-out cases at $\delta=30^{\circ}$ 


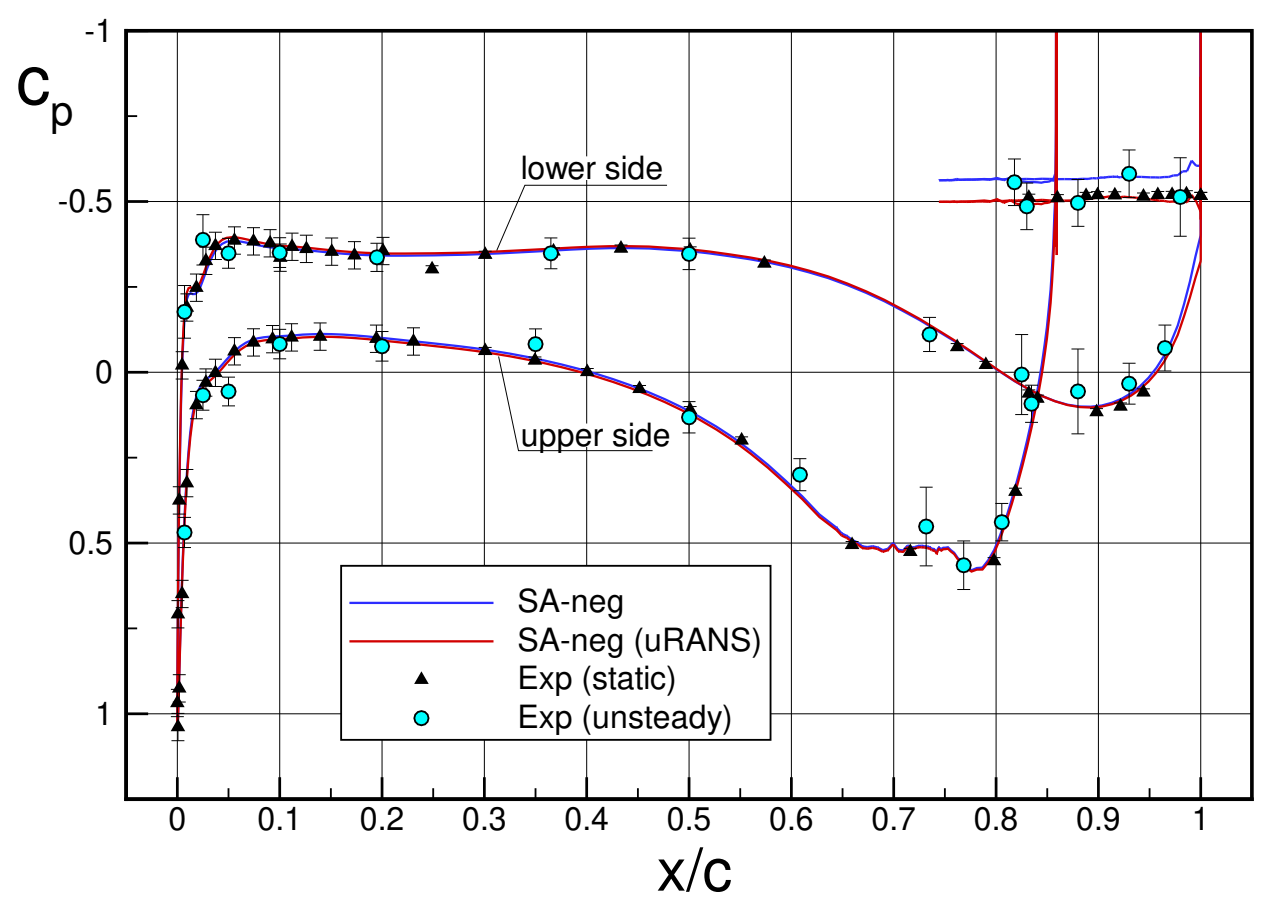

(a) $\alpha=0^{\circ}$.

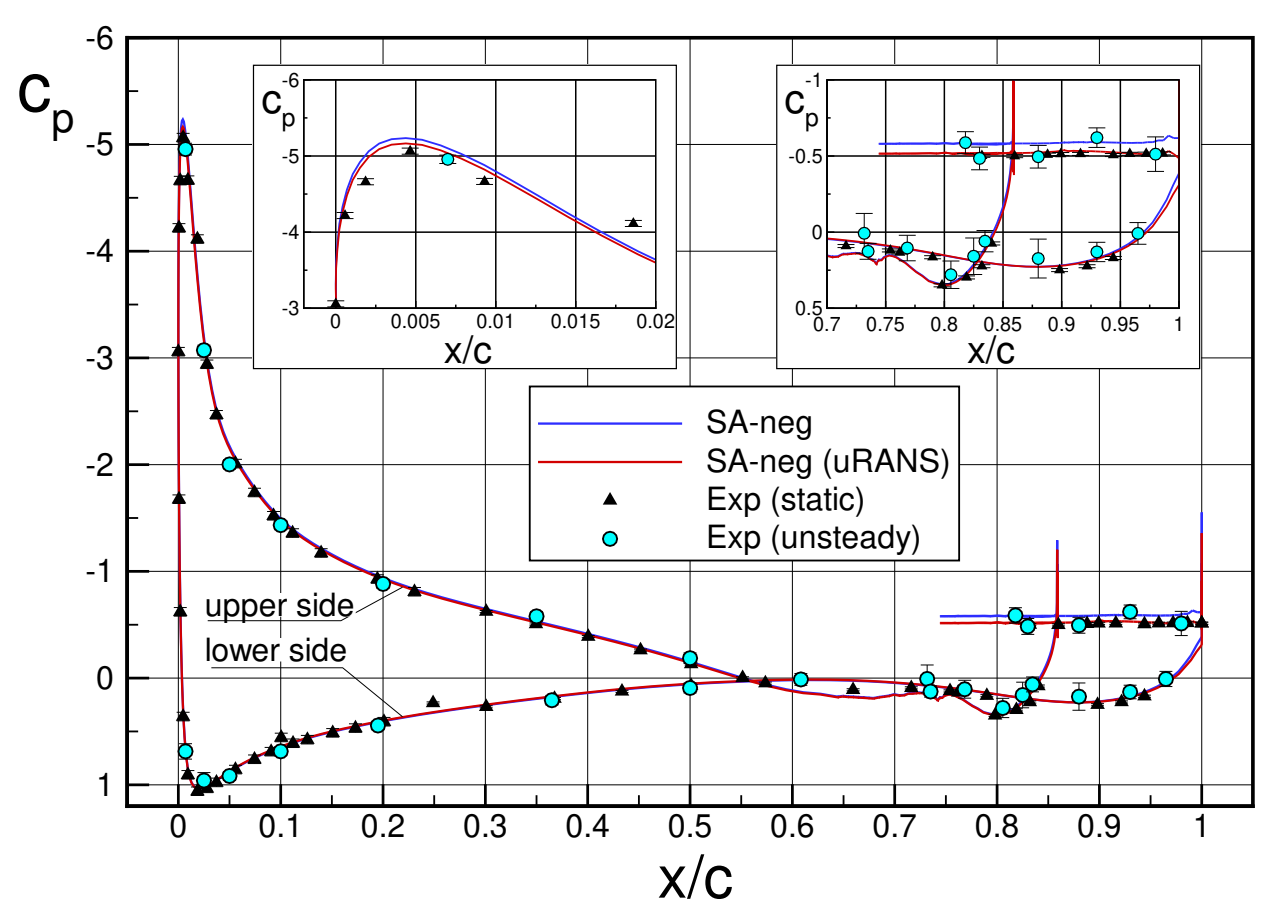

(b) $\alpha=10^{\circ}$.

Fig. 14 Influence of simulation approach (RANS vs. uRANS) on mean pressure distribution at $\delta=50^{\circ}$.

and $50^{\circ}$. The time step size for these computations was derived from a preliminary study of dynamic spoiler-out cases, in which it was observed that a temporal discretization of the moving spoiler in steps of $0.25^{\circ}$ per time step offered 
the best compromise between simulation accuracy, convergence behavior and wallclock time, see section V.A. Thus, depending on target spoiler deflection angle and deflection time, the time step size was adjusted such to resolve the spoiler motion in $0.25^{\circ}$-steps. The resulting time step sizes have then also been applied to the static spoiler-out cases, leading to non-dimensional time steps in the range of $0.05-0.06$, according to Eq. (5). Each simulation was run over a total physical time corresponding to 20 convective time units, i. e. $20 c / v_{\infty}$.

$$
\Delta t^{*}=\frac{v_{\infty} \Delta t}{c}
$$

Exemplary results for a spoiler deflected to $\delta=50^{\circ}$ at low and high angles of attack are shown in Fig. 14 In both cases, the time-accurate approach significantly improves the accuracy of the simulation in predicting the mean base pressure level behind the spoiler. At $\alpha=0^{\circ}$, switching from steady to unsteady simulation mode shifts curve by $\Delta c_{p}=0.06$ towards more positive pressure levels, leading to an excellent agreement with experimental data. It also becomes evident that the remaining parts of the pressure distribution remain unaffected. These observations also apply at $\alpha=10^{\circ}$. On top of that, as shown in Fig. 14(b), the uRANS simulation predicts a less pronounced suction peak at the leading edge, which also is in better agreement with experimental data.

Figure 15] shows the impact of the simulation approach on the mean flow topology in the vicinity of the spoiler. As mentioned before, the flow field is governed by a complex, three-dimensional interaction of vortices. It must be noted that Fig. 15 represents a slice through a 3D simulation result, hence potentially (and erroneously) suggesting that the flow field was purely two-dimensional, which clearly is not the case.

To be able to analyze the impact of the simulation approach on the resulting flow topology, the characteristic elements of the flow field have been numbered consecutively, starting from the pressure side of the spoiler towards the leading edge and beyond. As shown, all these elements are captured in both solutions. Their position and size, however, may differ significantly. For instance, the pressure-induced separation bubble at the spoiler hinge line, indicated by point 1 in Fig. 15, still is equally represented in both solutions. The same holds for the secondary spoiler vortex immediately aft of the spoiler, point 2 . In contrast, the primary spoiler vortex, point 3 , and its counterpart, the trailing edge vortex, point 4 , extend nearly $30 \%$ further downstream when using uRANS. This can easily be recognized by comparing the position of the downstream reattachment point, point 5. Due to this fact, these two vortices affect the upper surface pressure distribution aft of the spoiler to a lesser extent than it is the case in the steady RANS result. As a consequence, the typically induced low pressure region by these vortices aft of the spoiler is less pronounced when using uRANS, i. e. the base pressure is shifted towards more positive values of $c_{p}$ and, thus, being in better agreement with experimental data.

This once again highlights the main challenge when applying simulation tools to predict the aerodynamic behavior of spoilers: capturing the base pressure coefficient $c_{p b}$ aft of the spoiler as accurately as possible. Therefore, Fig. 16 

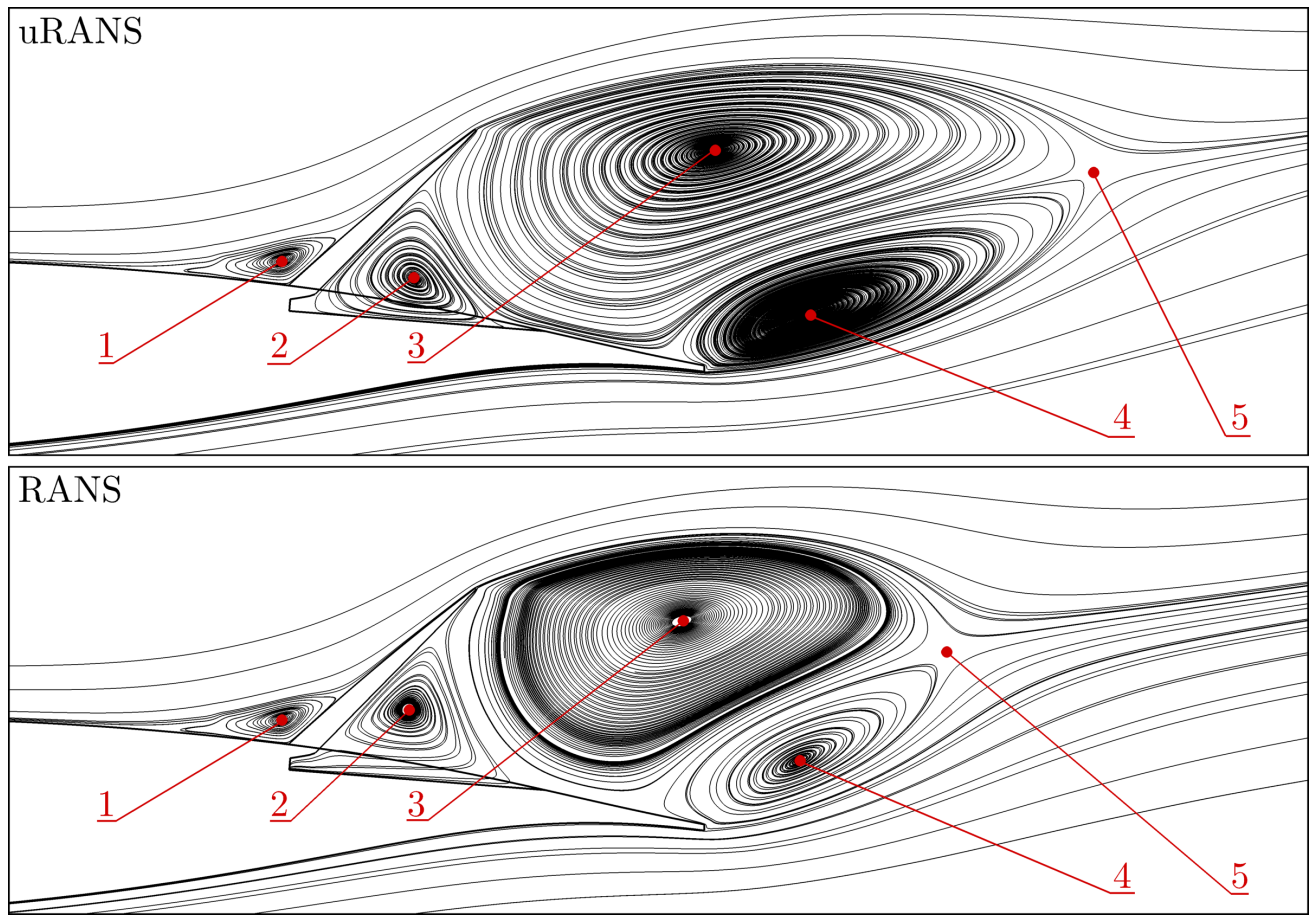

Fig. 15 Influence of simulation approach on mean flow topology at $\alpha=0^{\circ}$ and $\delta=50^{\circ}$.
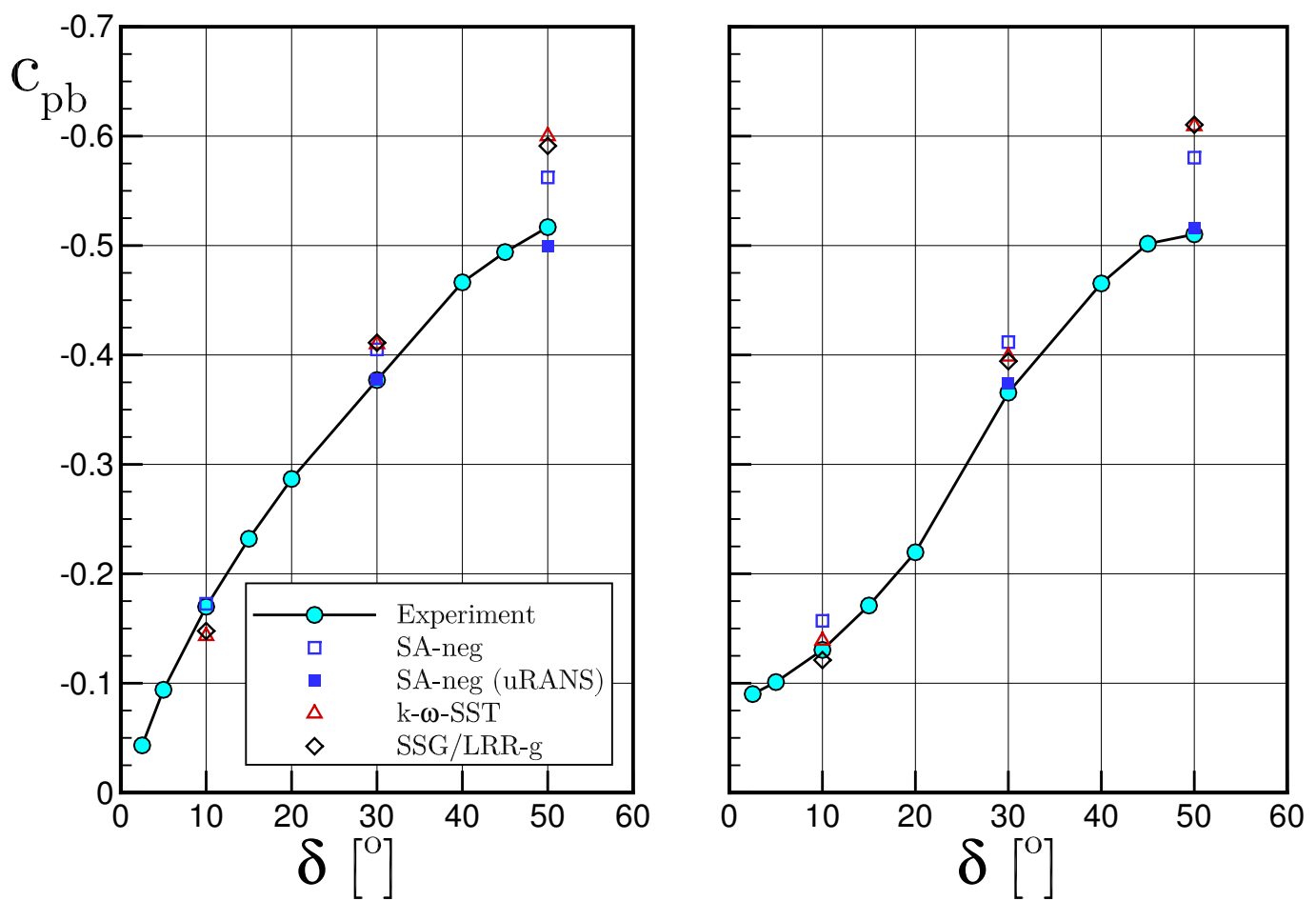

Fig. 16 Influence of turbulence model and simulation approach on resulting surface base pressure coefficient in the wake of the spoiler compared to experimental data at $\alpha=0^{\circ}$ (left) and $\alpha=10^{\circ}$ (right). 
summarizes the results so far on turbulence model and simulation approach influences in terms of accuracy in predicting the base pressure coefficient. In addition to the already known advantages of the SA-neg turbulence model in reproducing the experimental pressure distribution in general, see section IV.B. a further improvement in accuracy can be observed when switching from steady RANS (void blue squares) to time-accurate uRANS mode instead (filled blue squares), as shown for spoiler deflections of $\delta=30^{\circ}$ and $\delta=50^{\circ}$ at both low and high angles of attack.

In quantitative terms, for a spoiler deflected to $\delta=30^{\circ}$ at $\alpha=0^{\circ}$, the deviations between steady simulation and experiment, $\Delta c_{p b}=0.03$ or $7 \%$, could be completely eliminated when using uRANS. At $\delta=50^{\circ}$ and same angle of attack, the deviations were reduced from formerly $\Delta c_{p b}=0.29$ or $9 \%$ to $\Delta c_{p b}=0.02$ or below $4 \%$. At $\alpha=10^{\circ}$, the steady simulation using SA-neg yielded differences in the order of $\Delta c_{p b}=0.05-0.07$ or approx. 13\%. When using uRANS, these numbers could be reduced to $\Delta c_{p b}<0.01$ or below $2.2 \%$.

To summarize the influence of the simulation approach it can be stated that using uRANS leads to a significantly more accurate representation of the base pressure in the wake of statically deflected spoilers. However, this gain in accuracy in the presented cases came along with five times higher computational costs. Nonetheless, the validation results presented so far prove that, for the configuration and the onflow conditions investigated in this study, the chosen simulation approach is well-suited to predict the pressure distribution not only qualitatively, but also in quantitative terms for spoilers deflected up to $\delta=50^{\circ}$.

\section{Overset Grid Influence}

Before addressing dynamic spoiler simulations the influence of the gaps present in the overset grid was examined. To ensure a comparable spatial resolution the overset grid results were compared with the results produced with the fine one-block grid. A representative result for a spoiler deflected to $\delta=30^{\circ}$ is shown in Fig. 17 Two peaks marked with numbers "1" and "2" can be observed in the pressure distribution in Fig. 17(a) when using the overset grid, which are not present in case of the one-block grid. They indicate a sudden flow acceleration on both wing and spoiler (peak 1), just before the wing faces a recompression (peak 2). This behavior is caused by the flow through the spoiler hinge line gap, as shown in Fig. 17(b). The first peak is produced by the flow acceleration at the throat of the hinge line gap, whereas the recompression is caused by a kink of the wing geometry. In addition, a closer look at the base pressure region in Fig. 17(a) reveals that the overset grid slightly improves the results in that region. This effect, which is even more pronounced at $\delta=50^{\circ}$, is caused by the fact that the overset grid better resembles the experimental setup, where a small hinge line gap was present as well. Such a gap leads to a venting of the spoiler, effectively increasing the pressure aft of the spoiler. Hence, the low pressure region behind the spoiler is less pronounced than it is when using the one-block grid, where a similar venting is inhibited. Apart from these findings, no further grid influences were found. 


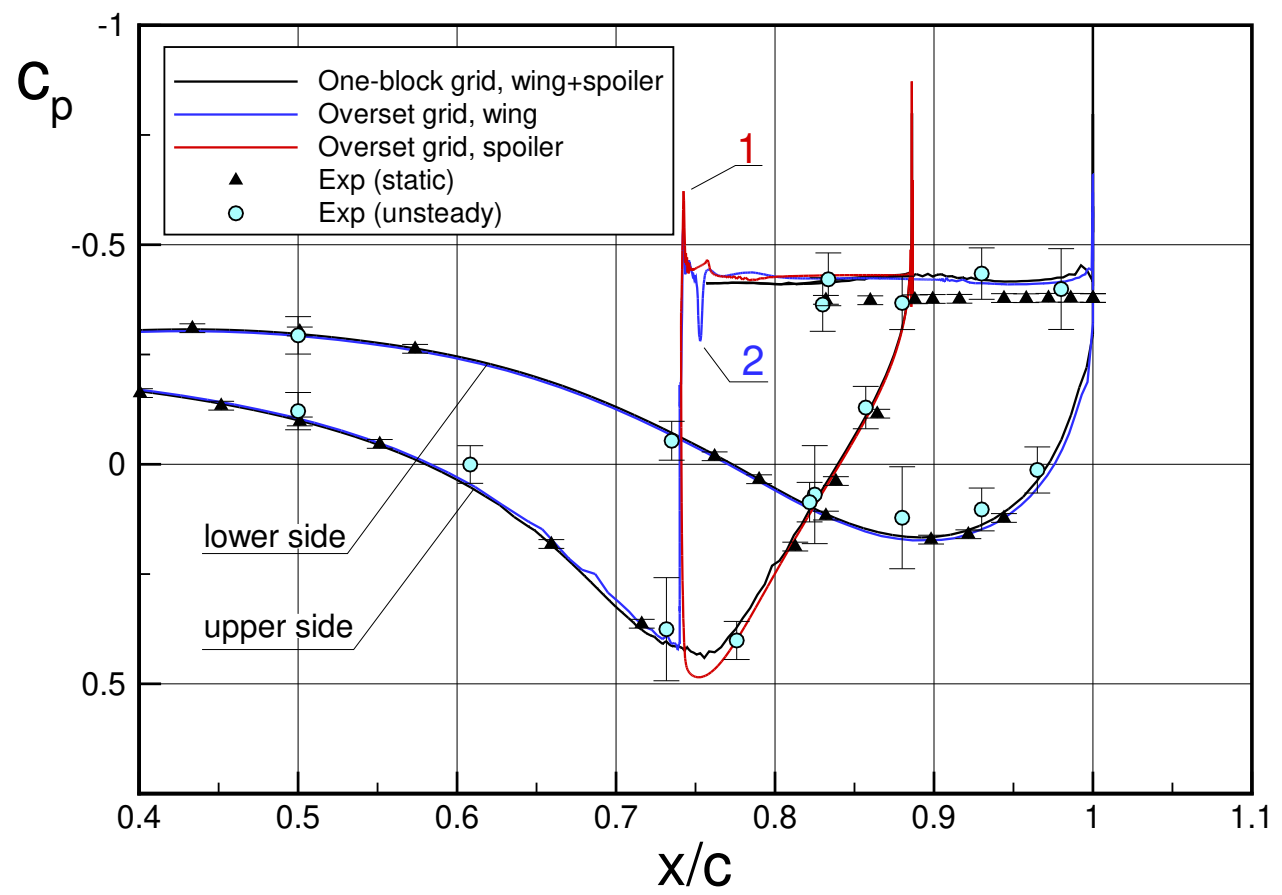

(a) Influence on pressure distribution at $\alpha=0^{\circ}$.

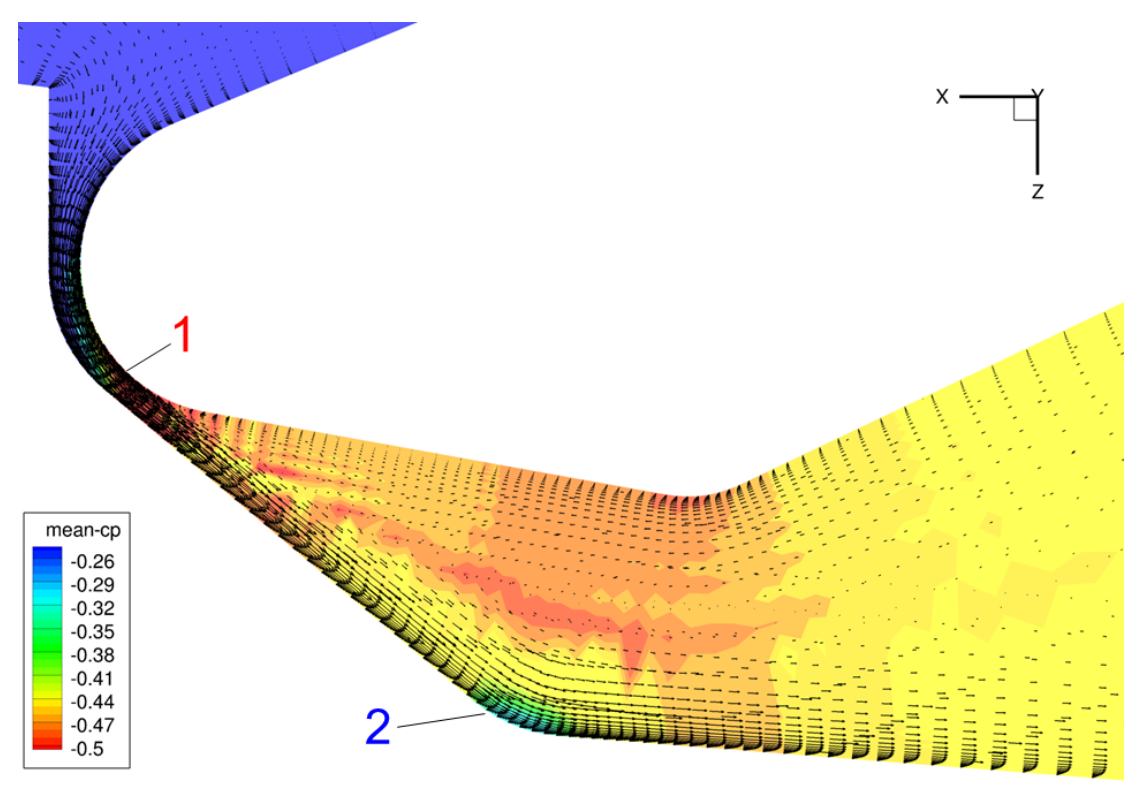

(b) Flow visualization in the spoiler hinge line gap region.

Fig. 17 Overset grid influence at $\delta=30^{\circ}$. 


\section{Dynamic Spoiler Deflections}

The numerical results presented in the following were obtained with the overset grid and the SA-neg turbulence model. The majority of the dynamic simulations was done for a target deflection angle of $\delta_{\text {target }}=30^{\circ}$, using the uRANS settings and the spoiler motion law according to section III

\section{A. Influence of Temporal Discretization}

Whenever dealing with time-accurate simulations, the time step $\Delta t$, with which the time is advanced in the simulation, needs to be chosen wisely. When investigating static geometries for instance, i. e. the geometry does not change in time, the usual procedure is to estimate an initial time step in advance, based on the frequency of occurring flow phenomena like vortices etc. Based on this initial time step, a time step study will usually be performed with the objective of identifying the largest time step (for computational reasons) which no longer alters the flow quantities of interest.

In the present context, the geometry does change in time. Therefore, in addition to the aforementioned aspect it needs to be taken into consideration how the motion of the spoiler shall be discretized in time. For spoiler simulations, this aspect is particularly important when the spoiler shall deflect from fully retracted state. If a too large time step was chosen, the robustness of the simulation might be affected adversely. In contrast, a too small one requires excessive computational resources. Hence, a time step sensitivity study was conducted to find a well-balanced time step with respect to robustness, convergence behavior and computational time.

Based on internal best practice guidelines for applying the overset grid technique in TAU the incremental spoiler motion should never exceed $\Delta \delta=0.4^{\circ}$ per $\Delta t$. Now, considering a spoiler deflection from $\delta=0 \rightarrow 30^{\circ}$ in $t_{d}=60 \mathrm{~ms}$, the maximum allowable time step with this constraint amounts to $(\Delta t)_{\max }=0.8 \mathrm{~ms}$. Based on that, three additional levels of refinement as summarized in Table 2 were investigated.

Table 2 Summary of relation between time step $\Delta t$, resulting angular discretization of the spoiler motion $\Delta \delta$, number of time steps required and computational time required to run a full spoiler deflection simulation from $\delta=0 \rightarrow 30^{\circ}$ in $t_{\mathbf{d}}=60 \mathrm{~ms}$ or $t_{\mathbf{d}}^{*}=6.9$.

\begin{tabular}{lccc}
\hline \hline$\Delta t$ & $\Delta \delta$ & No. of time steps & Computational time (normalized) \\
\hline $0.08 \mathrm{~ms}$ & $0.04^{\circ}$ & 740 & 4.7 \\
$0.254 \mathrm{~ms}$ & $0.125^{\circ}$ & 237 & 1.8 \\
$0.507 \mathrm{~ms}$ & $0.25^{\circ}$ & 119 & 1 \\
$0.8 \mathrm{~ms}$ & $0.4^{\circ}$ & 74 & 0.7 \\
\hline \hline
\end{tabular}

Figure 18 shows the aerodynamic response behavior for these time steps. The transient response largely remains unaffected by the chosen time step size. Only the smallest time step $\Delta t=0.08 \mathrm{~ms}$ leads to an oscillatory response in lift and pitching moment at $t / t_{\mathrm{d}}>0.7$. The mean value of the oscillations, however, is in good agreement with the other results, the oscillation amplitude remains small. Further small influences can be observed at the time of maximum adverse lift at $0.3<t / t_{\mathrm{d}}<0.4$, where a larger time step yields a more pronounced lift overshoot. Further analysis has 


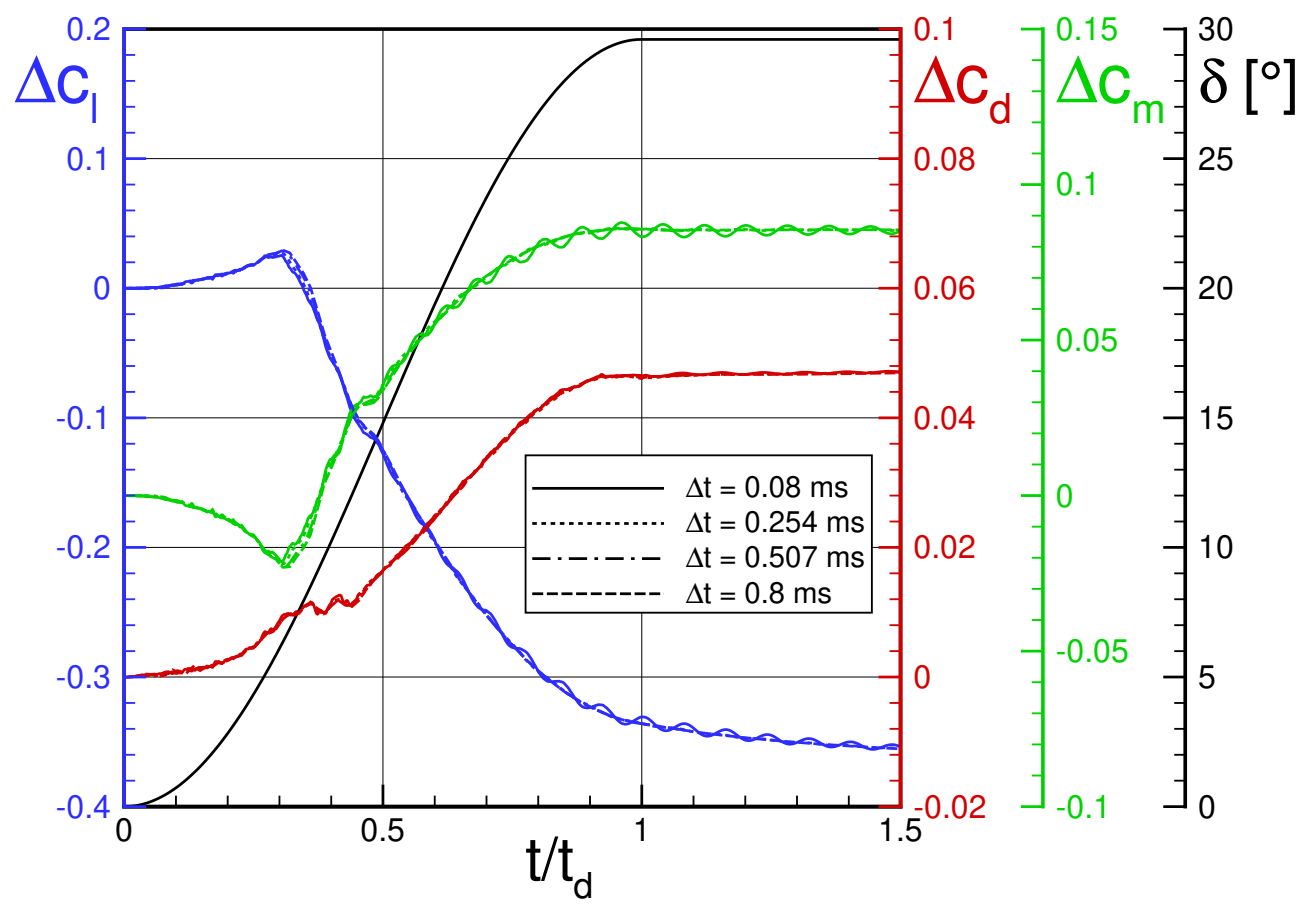

Fig. 18 Influence of time step size on transient aerodynamic response for a spoiler deflection from $\delta=0 \rightarrow 30^{\circ}$ in $t_{\mathbf{d}}=60 \mathrm{~ms}$ or $t_{\mathbf{d}}^{*}=6.9$.

shown that these deviations are much smaller than the scatter in the experimental data and, therefore, also considered small.

Altogether, the study revealed a minor influence only of the time step on the aerodynamic response due to a rapidly deflecting spoiler. Hence, simulation time and robustness turn into the primary criteria for choosing a time step for the subsequent validation studies. With that, the largest time step corresponding to an angular discretization of $0.4^{\circ}$ could have been used. However, in order to have some reserves in terms of robustness of the simulation, the next smaller time step was chosen, corresponding to an angular discretization of $0.25^{\circ}$. All subsequent simulations have been performed with a time step adjusted such to yield this exact angular discretization of the moving spoiler.

\section{B. Validation of Dynamic Spoiler Deployment Simulations}

Dynamic simulations from fully retracted state to target angles of $\delta=10^{\circ}, 30^{\circ}$ and $50^{\circ}$ as shown in Fig. 8 were performed. The intermediate angle of $30^{\circ}$ represents the core of the validation activities. For instance, the influence of the spoiler deflection time on the response has only been performed for this angle, whereas the simulations for the two remaining angles were only performed with the shortest deflection time. 


\section{Influence of Deflection Time for a Dynamic Deflection to $\delta=30^{\circ}$}

The following simulations were performed at $\alpha=0^{\circ}$ and varying deflection times, starting with a rather slow spoiler deflection of $t_{\mathrm{d}}^{*}=28.9$ down to a rapid deflection in $t_{\mathrm{d}}^{*}=6.9$. A summary of the investigated conditions is given in Table 3 .

Table 3 Variation of deflection time for a spoiler deflecting from $\delta=0 \rightarrow 30^{\circ}$ at $M=0.2$ and $\alpha=0^{\circ}$.

\begin{tabular}{lccc}
\hline \hline Case & $\begin{array}{c}\text { Deflection time } \\
t_{\mathrm{d}}\end{array}$ & $\begin{array}{c}\text { Non-dimensional deflection time } \\
t_{\mathrm{d}}^{*}(\text { Eq. (1) })\end{array}$ & $\begin{array}{c}\text { Resulting mean } \\
\text { deflection rate }\end{array}$ \\
\hline Slow & $250 \mathrm{~ms}$ & 28.9 & $120^{\circ} \mathrm{s}^{-1}$ \\
Intermediate & $125 \mathrm{~ms}$ & 14.5 & $240^{\circ} \mathrm{s}^{-1}$ \\
Rapid & $60 \mathrm{~ms}$ & 6.9 & $500^{\circ} \mathrm{s}^{-1}$ \\
\hline \hline
\end{tabular}

The following validation relies on a comparison of the change in sectional force and moment coefficients over time due to the deployment of the spoiler. In order to not only compare the data qualitatively, but also in a quantitative manner, the following characteristic parameters according to Fig. 1 are analyzed in more detail:

- the initial lift overshoot, also known as adverse lift, $\Delta c_{l, \text { adv }}$ and its associated moment in time $t_{\mathrm{adv}}$,

- the delay time $t_{\text {delay }}$, until the desired reaction of the spoiler (lift reduction) sets in,

- the final change of force and moment coefficients, e. g. $\Delta c_{l \text {,final }}$, which has been evaluated after $2 t_{\mathrm{d}}$.

Figure 19 shows a comparison between simulation and experiment for a slow spoiler deflection to $\delta=30^{\circ}$ in $t_{\mathrm{d}}^{*}=28.9$. As mentioned in section $\left[\right.$ no adverse lift is to be expected if $t_{\mathrm{d}}^{*}>5-9$. This general observation by Mabey and others is reflected in the experimental data and also reproduced by the simulation.

Looking at the transient lift response first, another characteristic of spoiler aerodynamics becomes evident: there always is a time delay between motion onset and the onset of the lift reduction. The desired lift reduction does not set in until the delay time $t_{\text {delay }}$, which depends on the deflection time, has passed by. Experimental data suggest this to happen at $t / t_{\text {delay }}=0.21$, whereas the simulation predicts it at $t / t_{\mathrm{d}}=0.25$. At this instant in time, the spoiler has reached an instantaneous deflection angle of $\delta=3.1^{\circ}$ in the experiments and of $\delta=4.4^{\circ}$ in the simulation. At this deflection angle the flow separating at the spoiler tip still reattaches just before reaching the trailing edge. Exceeding this critical angle will lead to a full flow separation and wake development aft of the spoiler, which results in a loss of lift. An interaction with the flow past the undisturbed lower side of the airfoil sets in, ultimately resulting in the complex flow topology depicted in Fig. 15. In the following, the simulation result matches the experimental data very well and mostly is within or at the edge of the experimental scatter until the end of the simulation at $t / t_{\mathrm{d}}=2$. The final lift reduction $\Delta c_{l \text {,final }}$ is predicted by the simulation with an accuracy of over $99 \%$.

The drag response is fully within experimental scatter from the beginning to the end of the simulation. The final drag reduction at $t / t_{\mathrm{d}}=2$ is also predicted by the simulation at an accuracy of over $99 \%$. In contrast to the transient lift 


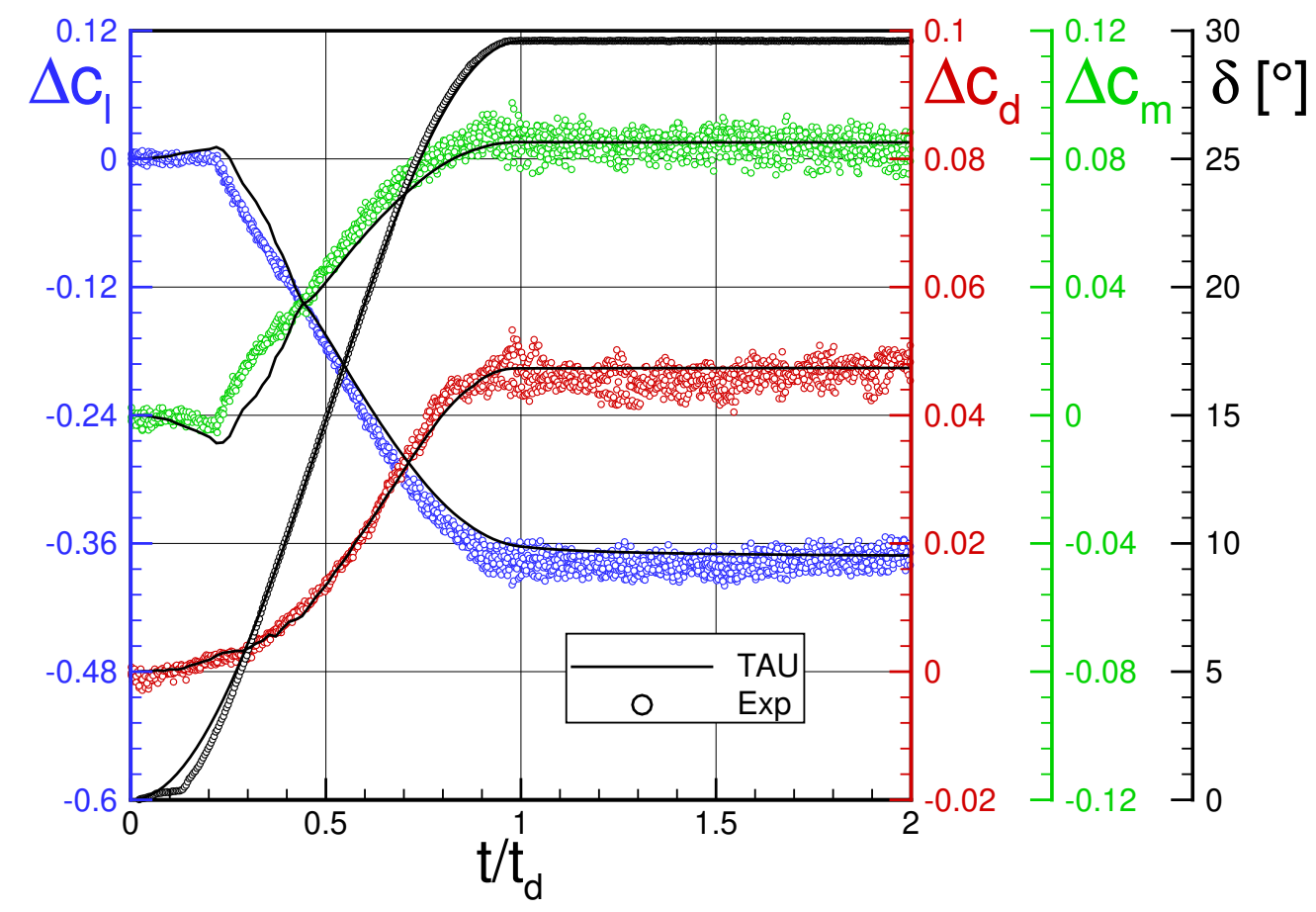

Fig. 19 Aerodynamic response for a slow spoiler deflection from $\delta=0 \rightarrow 30^{\circ}$ in $t_{\mathbf{d}}=250 \mathrm{~ms}$ or $t_{\mathbf{d}}^{*}=28.9$.

response, the drag coefficient does not feature a pronounced delay time. Additional drag is immediately produced once the spoiler starts to move. This is due to the fact that, once the spoiler deflects from its retracted state, the rising tip of the spoiler acts as a geometric discontinuity in the contour of the airfoil. This always leads to flow separation, which in turn increases drag almost instantly. Lift, in contrast, is not changed until the overall circulation around the airfoil is altered. As mentioned above, this only happens when the wake aft of the spoiler extends beyond the trailing edge.

By and large, the observations for the lift response also hold for the pitching moment. Only at $t / t_{\mathrm{d}}=0.25$, an overshoot in pitching moment can be recognized by the simulation, together with a slightly delayed pitching response. The final change in pitching moment is reproduced with the same accuracy again as before.

Figure 20 shows the aerodynamic response for an intermediate spoiler deflection to $\delta=30^{\circ}$ in $t_{\mathrm{d}}^{*}=14.5$. The observations from the slow deflection mostly hold for this case as well due to the fact that the non-dimensional deployment time still is above the critical range of $t_{\mathrm{d}}^{*}=5-9$. In contrast to earlier observations by other researchers, the experimental data at $0.2<t / t_{\mathrm{d}}<0.3$ indicate that the typical adverse unsteady effects, in particular the overshoot in lift and, even more so, the undershoot in pitching moment already start to build up at this intermediate deflection. This important observation is reproduced well by the simulation in qualitative terms and the amplitudes of the over-/undershoots are also in very good quantitative agreement. However, the associated peaks are not as clearly pronounced in the simulation. For instance, the build-up of lift overshoot and pitching moment undershoot starts much earlier in the simulation and more gradual, almost right after onset of the spoiler motion. In contrast, in the experiments, no change 


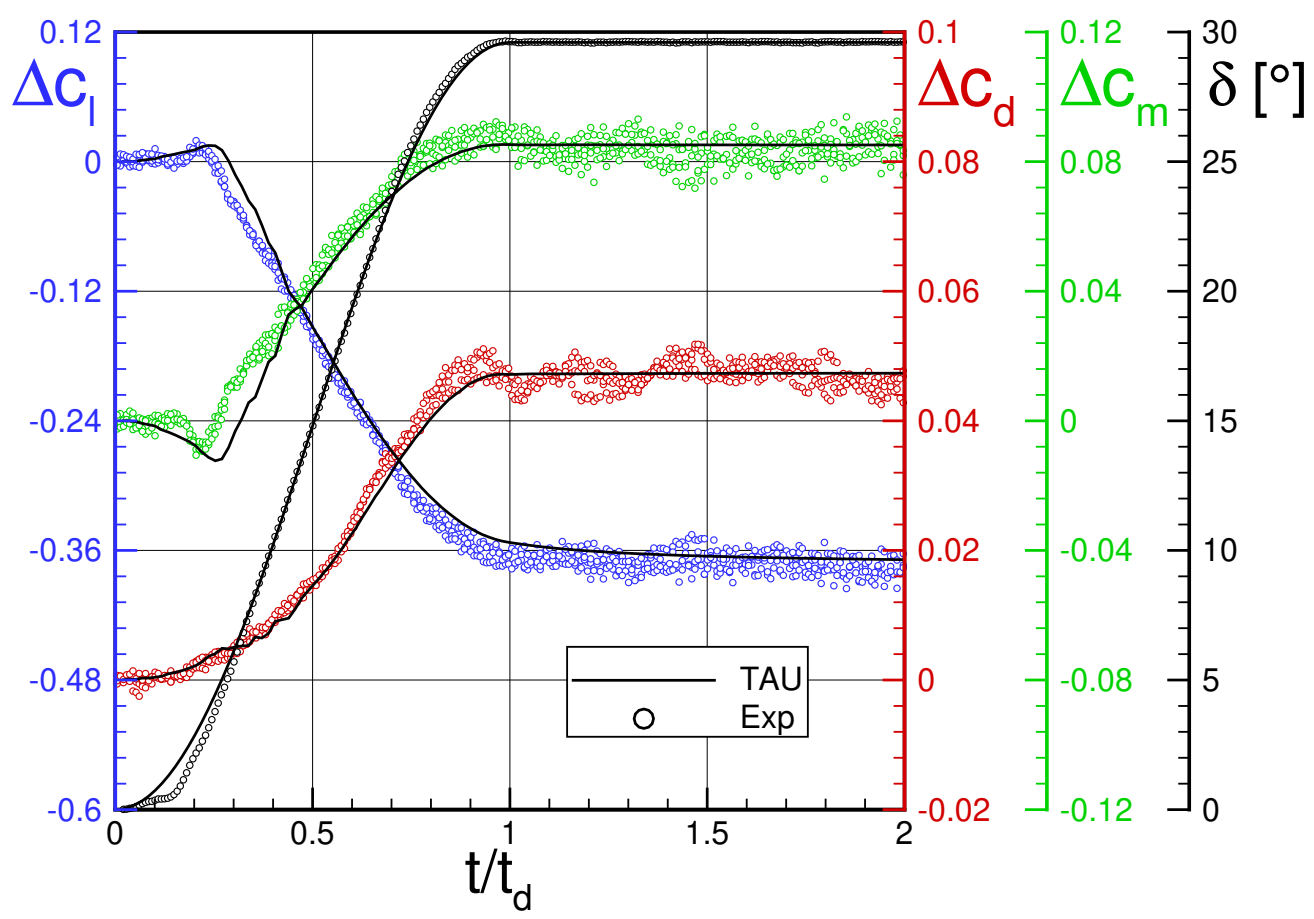

Fig. 20 Aerodynamic response for an intermediate spoiler deflection from $\delta=0 \rightarrow 30^{\circ}$ in $t_{\mathbf{d}}=125 \mathrm{~ms}$ or $t_{\mathbf{d}}^{*}=14.5$.

in lift and pitching moment is observed until $t / t_{\mathrm{d}}<0.15$. A more detailed analysis has revealed that the observed differences between simulation and experiment until $t / t_{\mathrm{d}}<0.35$ are caused by deviations in the corresponding spoiler deflection angle over time curves, as illustrated by the $\delta$-curves in Figure 20. While the spoiler in the simulation is moved according to the 1-cos motion law given by Eqs. (3) and (4), i. e. the motion sets in right at the beginning of the simulation, the experimental data revealed no motion at all within the first $15 \%$ of the deflection sequence. The reason for that consists in mechanical play in the kinematics of the wind tunnel model. This in turn means that the spoiler in the experiments has completed its motion from fully retracted state to its end position of $\delta=30^{\circ}$ in only $85 \%$ of the nominal deflection time. Hence, the effective mean deflection rate is higher than in the simulation or, in other words, the effective non-dimensional deflection time is shorter, with $t_{\mathrm{d} \text {,effective }}^{*}=12.2$ compared to 14.5 in the simulation. As a consequence, the unsteady effects are more pronounced in the experimental data. The remainder of the curves is similar to the behavior at the slow deflection sequence, i. e. the simulation predicts a response behavior that is largely within the experimental scatter until the spoiler has reached its end position and transient effects have faded out.

The result of a deflection sequence with further reduced deflection time, the spoiler now deflects rapidly within $t_{\mathrm{d}}^{*}=6.9$, is illustrated in Fig. 21 . In this case, significant unsteady effects are to be expected according to theory. The amplitudes of the under-/overshoots observed in the response of lift and pitching moment at $0.2<t / t_{\mathrm{d}}<0.4$ indeed are 2-3x higher than in the intermediate deflection sequence. In addition, the adverse unsteady effects occur later and the 


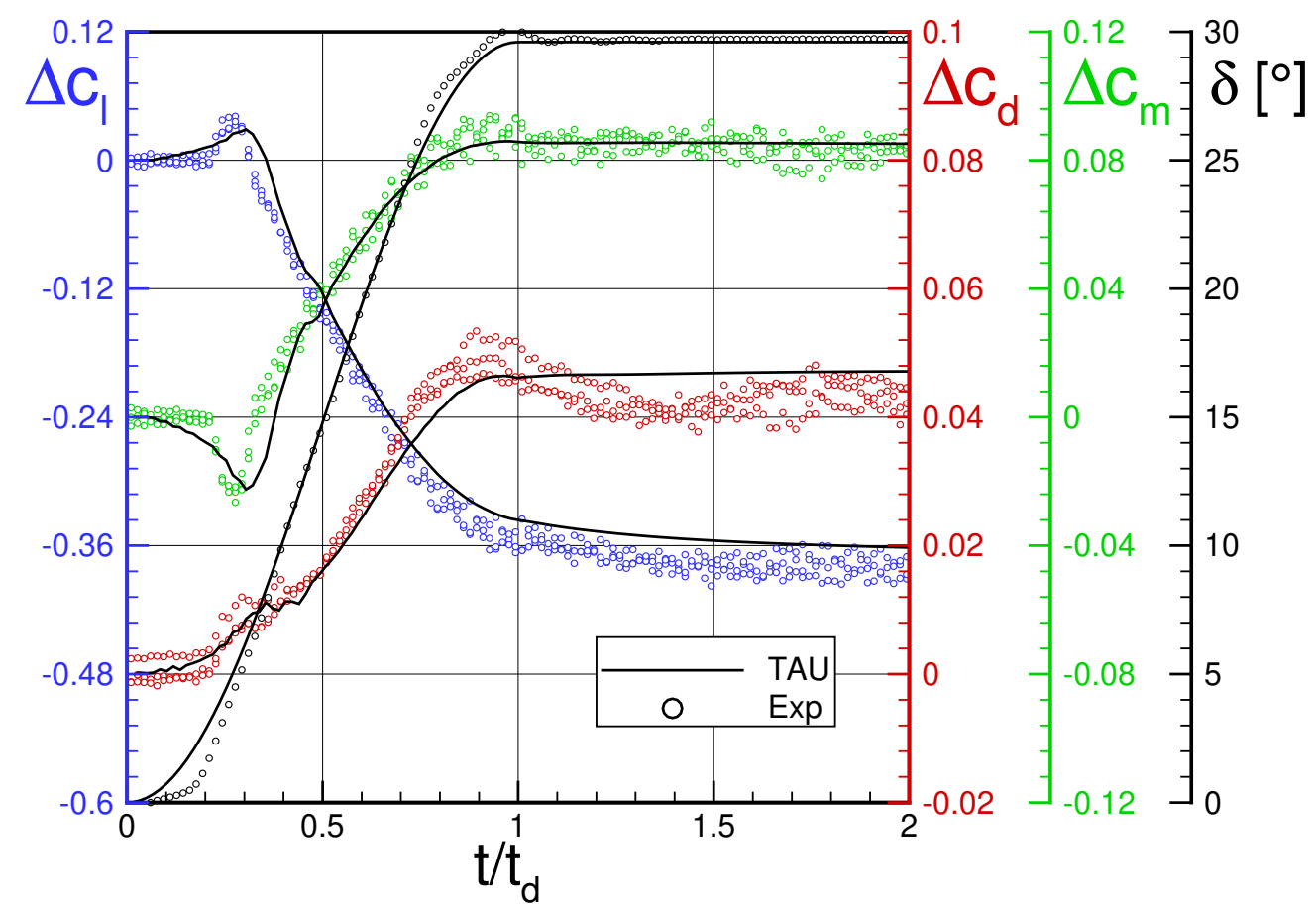

Fig. 21 Aerodynamic response for a rapid spoiler deflection from $\delta=0 \rightarrow 30^{\circ}$ in $t_{\mathbf{d}}=60 \mathrm{~ms}$ or $t_{\mathbf{d}}^{*}=6.9$.

delay time is increased as well. Qualitatively, these effects are well captured by the simulation.

Quantitative differences, however, remain in the lift response. For instance, the maximum lift overshoot as predicted by the simulation amounts to $\Delta c_{l, \text { adv, Sim }}=0.029$, whereas experimental data suggests a mean value of $\Delta c_{l, \text { adv,Exp }}=0.037$ or $28 \%$ higher than in the simulation. The associated moment in time, $t_{\mathrm{adv}}$, occurs at $30 \%$ of the deflection time in the simulation and at $28 \%$ in the experiments. The delay time in the simulation is also higher than in the experiments (35\% vs. $31 \%$ of deflection time), which is consistent to the findings of the two aforementioned cases. The final change in lift at the end of the simulation is predicted by the simulation with an accuracy of $95 \%$. The drag response remains almost entirely within the experimental scatter, although the final change in drag is overpredicted by the simulation by $11 \%$. The simulated initial pitching moment undershoot $\Delta c_{m}$,adv deviates by $5 \%$ from the experimental value and again happens a little later in the simulation ( $30 \%$ vs. $28 \%$ of deflection time). Consequently, the same holds for the delay time (38\% vs. $32 \%$ ). The deviations observed in the pitching moment response within the first $40 \%$ of the deflection sequence are most probably attributed to the already discussed differences in the spoiler angle curves due to mechanical play. From $0.4<t / t_{\mathrm{d}}<2$ the simulation result nearly coincides with the mean value of the experimental data. The final change in pitching moment is accurately reproduced by the simulation.

A summary of the characteristic unsteady effects is given in Table 4. The amplitude $\Delta c_{1, \text { adv }}$ nonlinearly increases the shorter the deployment time, reaching a maximum overshoot of nearly $8 \%$ of the final lift reduction in the respective case. It also becomes evident that the characteristic times, like the time at which the adverse lift occurs, $t_{\text {adv }}$, or the 
Table 4 Summary of adverse lift and time delay characteristics for spoiler deployments to $\delta=30^{\circ}$ at $\alpha=0^{\circ}$.

\begin{tabular}{lcccc}
\hline \hline Deployment time & $\Delta c_{1, \text { adv }}$ & $\Delta c_{1, \text { adv }} / \Delta c_{1, \text { final }}$ & $t_{\text {adv }} / t_{\mathrm{d}}$ & $t_{\text {delay }} / t_{\mathrm{d}}$ \\
\hline$t_{\mathrm{d}}^{*}=28.6$ & 0.0113 & $3.0 \%$ & 0.220 & 0.255 \\
$t_{\mathrm{d}}^{*}=14.3$ & 0.0152 & $4.1 \%$ & 0.237 & 0.288 \\
$t_{\mathrm{d}}^{*}=6.9$ & 0.0290 & $7.8 \%$ & 0.304 & 0.357 \\
\hline \hline
\end{tabular}

instant in time at which the desired spoiler reaction first sets in, $t_{\text {delay }}$, both increase the shorter the deflection time or the higher the deflection rate. This is especially important to consider for applications like gust load alleviation where control surfaces like spoilers are deflected at high rates to counteract the effects of atmospheric disturbances. The data in Table 4 clearly underlines that, even for the slowest deflection sequence, over $25 \%$ of the deployment time has already passed by until the reduction in lift finally sets in. For the fastest deployment sequence, this value even increases to $35 \%$.

To summarize the validation efforts for this exemplary application of a deflection sequence to $\delta=30^{\circ}$ at varying deflection times, it can be stated that the applied simulation approach is capable of reproducing all the relevant characteristic dynamic effects from a qualitative point of view. Quantitatively, the characteristic times as predicted by the simulation lag behind the actual times from the experiments. Moreover, the overshoots in the simulation appear less sharp or less clearly pronounced, which is most probably due to differences in the actual spoiler position over time. This aspect will be further investigated in the future. Despite the observed quantitative differences in predicting the characteristic times, the amplitude of the unsteady effects and the general transient behavior until the end of the simulation have largely been in very close agreement to experimental data. Therefore, this first validation step for dynamic spoiler simulations with the DLR TAU code is considered to be successfully accomplished.

\section{Influence of Deflection Angle}

As mentioned before, the case of a deflection to $\delta=30^{\circ}$ represents the core of the validation activities. Nonetheless, it has also been investigated how the simulations perform when the spoiler deflects to a smaller or larger angle. In the following, results for deflection sequences to $\delta=10^{\circ}$ and $50^{\circ}$, each with the shortest deflection time, i. e. highest deflection rate, are presented at $\alpha=0^{\circ}$.

Figure 22 shows the response to a rapid deflection to $10^{\circ}$ in $t_{\mathrm{d}}^{*}=6.9$ or $60 \mathrm{~ms}$, resulting in a mean rate of deflection of $167^{\circ} \mathrm{s}^{-1}$. Using the same axis scale as before, it can easily be noted that the unsteady effects are much weaker compared to the deployment to $\delta=30^{\circ}$ at the same non-dimensional deployment time, Fig. 21. For instance, the maximum lift overshoot $\Delta c_{l, \text { adv }}$ has nearly halved, it also occurs later at $t / t_{\mathrm{d}}=0.4$ compared to $t / t_{\mathrm{d}}=0.3$ before. However, more pronounced differences in the time history of the deflection angle can be observed for $t / t_{\mathrm{d}}<0.35$ and have to be taken into consideration when putting these results into perspective. Until $t / t_{\mathrm{d}}<0.25$, the spoiler effectively deflects faster in the simulation than in the experiments. Beyond this instant in time, the experimental data displays a much stronger gradient until the spoiler reaches its end position. As mentioned before, the deviations between the simulated spoiler 


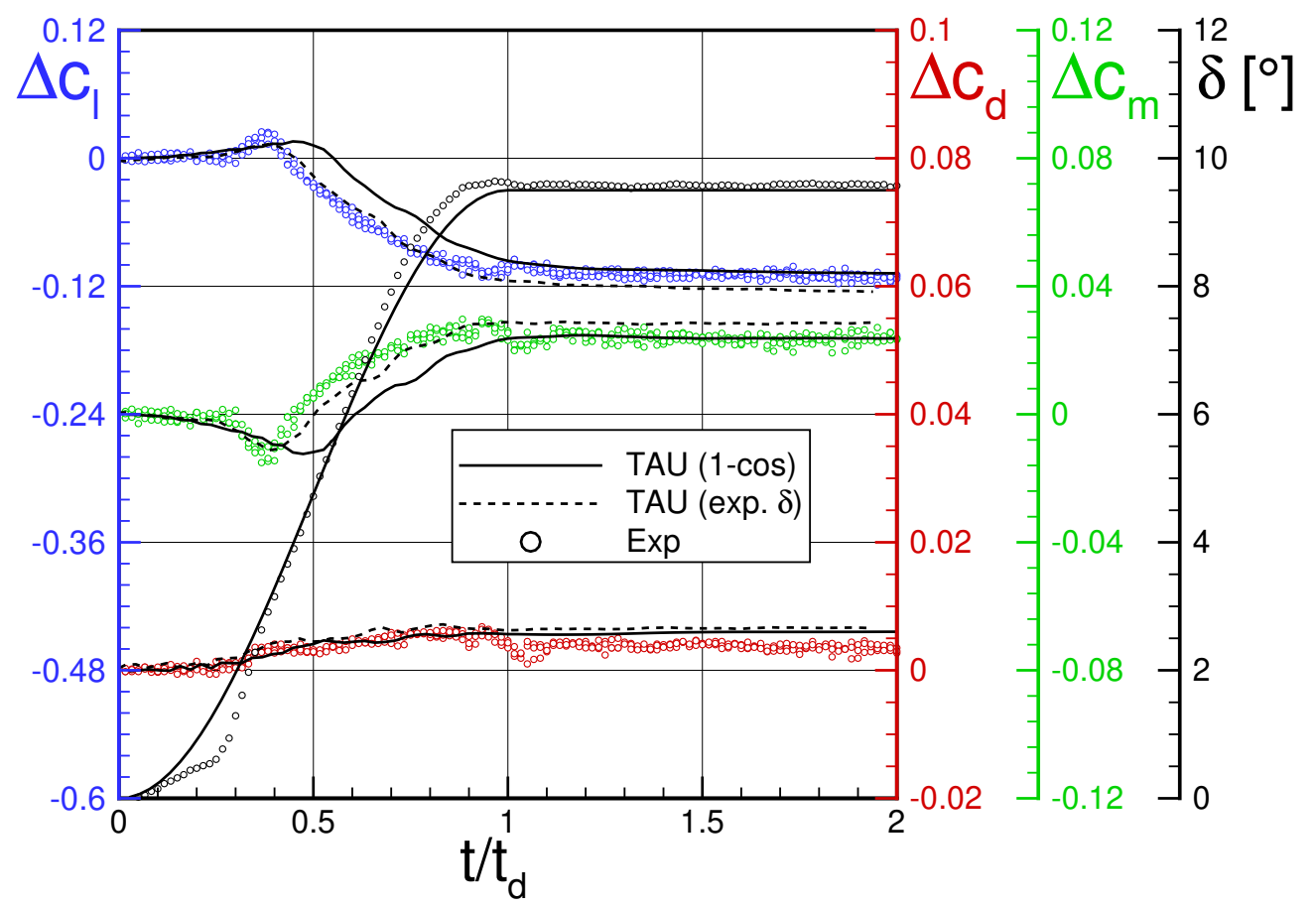

Fig. 22 Aerodynamic response for a rapid spoiler deflection from $\delta=0 \rightarrow 10^{\circ}$ in $t_{\mathbf{d}}=60 \mathrm{~ms}$ or $t_{\mathbf{d}}^{*}=6.9$.

motion over time and the synchronized, actual motion in the experiments are due to mechanical play. Consequently, the spoiler in the simulation performs a smooth motion with gradually increasing spoiler angle, whereas in the experiments, the spoiler rather performs a step-type motion with locally very different deflection rates. Although a more profound analysis into these findings still is subject to ongoing work, it is expected that the deviations in the time history of the spoiler angle within the first $20 \%-30 \%$ of the sequence cause the more pronounced unsteady effects in the experimental data.

A first proof of this hypothesis is also shown by the dashed curve in Fig. 22, which represents a simulation result in which the spoiler had not been moved according to the motion law given by Eqs. (3) and (4), but according to the actual time history of the spoiler deflection angle as recorded in the experiments instead. It becomes evident that almost all of the initial differences between simulation and experiment vanish as soon as the spoiler in the simulation is moved according to its experimental time history. It has to be pointed out that most simulations in this study had been performed well before any wind tunnel data was available. Therefore, the actual spoiler time history was only exemplarily used in the simulation.

Figure 23 shows the response to a rapid deflection to $50^{\circ}$ in $t_{\mathrm{d}}^{*}=9.3$ or $80 \mathrm{~ms}$, resulting in a mean rate of deflection of $625^{\circ} \mathrm{s}^{-1}$. First of all, it can be noted that the experimental time history of the spoiler angle now is in good agreement with the simulated curve. In contrast to the previous case, there is no need to recompute this sequence with the experimental spoiler time history as input to the simulation. It also becomes evident that the unsteady effects in lift and 


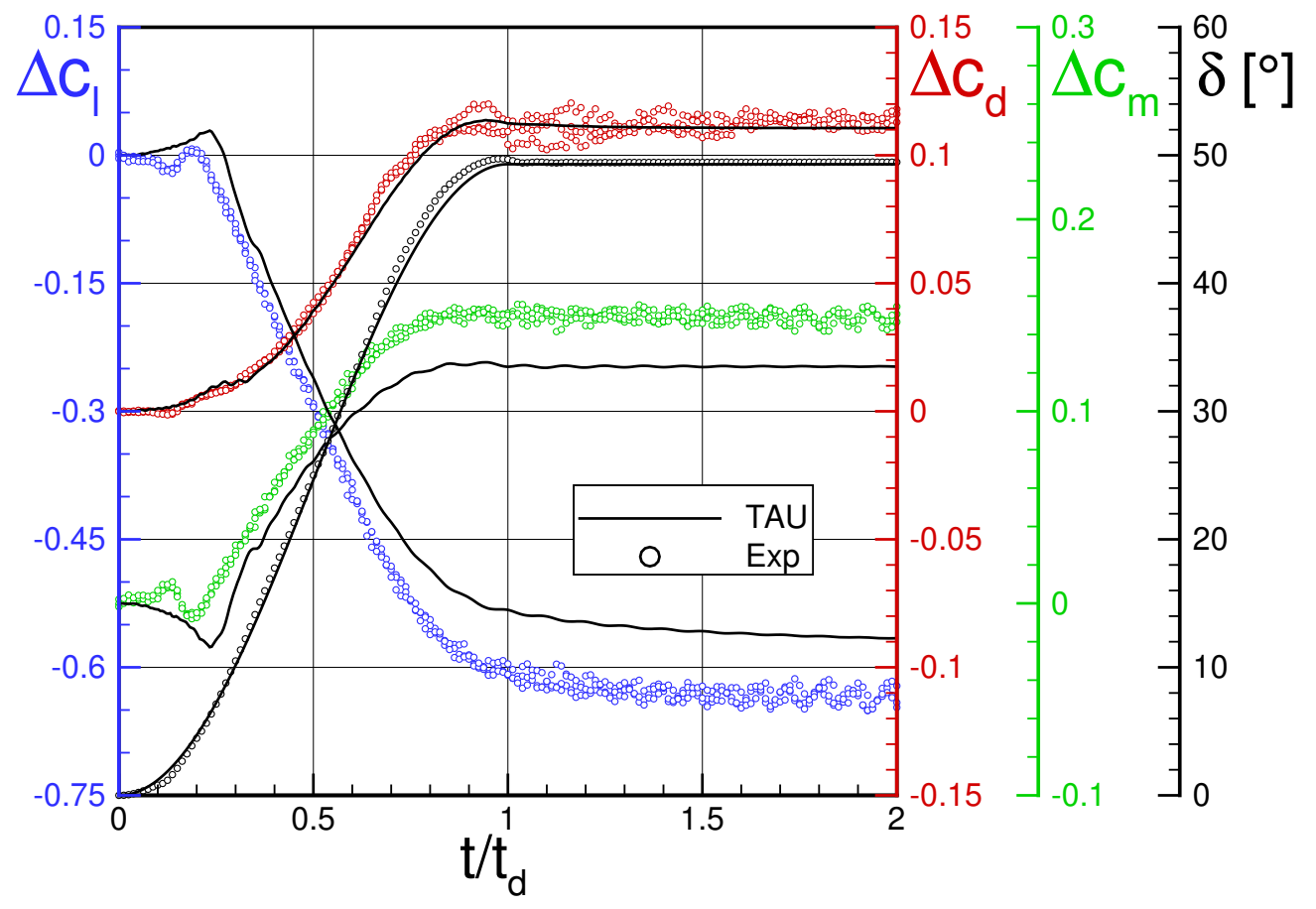

Fig. 23 Aerodynamic response for a rapid spoiler deflection from $\delta=0 \rightarrow 50^{\circ}$ in $t_{\mathbf{d}}=80 \mathrm{~ms}$ or $t_{\mathbf{d}}^{*}=9.3$.

pitching moment response are much smaller than before, which according to theory, is due to the higher non-dimensional deployment time in this case $\left(t_{\mathrm{d}}^{*}=9.3\right.$ vs. $t_{\mathrm{d}}^{*}=6.9$ before $)$. Looking at the initial lift response at $0.1<t / t_{\mathrm{d}}<0.2$, for the first time a small undershoot can be recognized in the experimental data, which is not present in the simulation. The reason for that is not yet known. The remaining lift response is predicted well by the simulation in a qualitative sense, but the delay time, for instance, lags behind the experimental value by six percentage points. This observation, however, is consistent with the findings in all other cases. The final lift reduction at $t / t_{\mathrm{d}}<2$ is underestimated by the simulation by $13 \%$. These findings also hold for the pitching moment response, except for the fact that the final change in pitching moment is underestimated by $20 \%$. The drag response, on contrast, perfectly matches the experimental data, the simulated curve always is within the scatter of the experimental data. The final change in drag is slightly underestimated by the simulation by $2.5 \%$.

To summarize the validation work for spoilers deflecting rapidly to $10^{\circ}$ and $50^{\circ}$ it can be stated that the simulation again is well capable of predicting the aerodynamic response in a qualitative sense. In addition, if the actual spoiler time history as recorded in the experiments is used as input for the simulation, the simulation results also are in good quantitative agreement with experimental data. Only when deflecting to $\delta=50^{\circ}$, differences between simulation and experiment in predicting the final lift and moment response exceed $10 \%$. The characteristic times as computed by the simulation consistently lag behind the experimental ones, i. e. the simulation reacts more sluggishly to the deflecting spoiler. One reason for that might be that the dynamics of the vortex creation aft of the spoiler and the subsequent 
interaction of those vortices simply cannot be captured more accurately either by the turbulence model or by the entire simulation approach. Further analyses into this aspect will follow.

Finally, a comparison of the unsteady effects at the beginning of the deflection sequences reveals that they are much more dominant when deflecting to $\delta=30^{\circ}$ than to $10^{\circ}$, although the respective non-dimensional deployment times were identical with $t_{\mathrm{d}}^{*}=6.9$. This in turn suggests that the non-dimensional deployment time $t_{\mathrm{d}}^{*}$ might well be a good indicator to determine if these unsteady effects will occur, as stated by theory. However, this parameter alone does not allow for any conclusions on the strength of these effects. Therefore, further research work on this topic will be conducted by DLR to develop a better understanding on the physical mechanisms driving these effects.

\section{Conclusion and Outlook}

Results of an extensive and still ongoing verification and validation campaign of the DLR TAU RANS flow solver for spoiler applications were presented in this work. The influence of static and dynamic spoiler deflections was numerically investigated at low speeds and compared to experimental data. Starting with static spoiler deflections up to $50^{\circ}$, typical influential parameters, such as turbulence model, spatial discretization, grid type and the influence of a time-accurate simulation approach were assessed. It turned out that the initially chosen steady simulation approach, using unstructured grids and the SA-neg turbulence model, already is well capable of reproducing the pressure distribution. However, deficiencies remained, especially in accurately predicting the base pressure aft of the deployed spoiler. All turbulence models tended to overestimate the low pressure in that region. It could be shown that these deficiencies could be significantly reduced within the entire range of investigated angles of attack and spoiler deployment angles if the simulation was performed time-accurately (uRANS). In the present cases, this gain in accuracy, however, came at 4-5x higher computational costs. Based on the encouraging results for static deflections, dynamic, ramp-type deployment sequences were investigated. It was shown that the aerodynamic response was in very good agreement with experimental data for the cases up to $\delta=30^{\circ}$, even for the fastest deflections. Whenever differences between simulation and experiment in the transient phase were identified, they could be traced back to a nonconforming time history of the spoiler motion in simulation and experiment. At $\delta=50^{\circ}$, however, more pronounced differences were observed in the lift and pitching moment responses which could no longer be attributed to a potentially nonconforming spoiler motion. A more profound analysis into the reasons for that are under work. The same holds for simulating dynamic deflections at higher angles of attack. So far, all dynamic cases were computed at $\alpha=0^{\circ}$ only.

In summary, the presented validation efforts of the numerical approach have been considered successful so far. This work will continue, for instance, to determine which parameters exactly govern the amplitude of the adverse dynamic effects. Moreover, the effects of wing sweep as well as Mach and Reynolds numbers up to flight conditions will be investigated. Once accomplished, the simulation approach will be utilized to develop future, more efficient load alleviation functionalities. 


\section{Acknowledgments}

The present work was part of the internal DLR research projects Mephisto and Diabolo, which were both supported by the German Ministry of Defence. The author would like to thank his colleagues Jochen Wild and Andreas Schütte from the DLR Institute of Aerodynamics and Flow Technology for their continuous support and fruitful discussions while conducting this work. The author would also like to thank Gunnar Einarsson and Frank Spiering, same institute, for providing technical assistance in using the TAU flow solver whenever needed.

\section{References}

[1] Sensburg, O., Becker, J., Lusebrink, H., and Weiss, F., “Gust Load Alleviation on Airbus A300,” ICAS paper 82-2.2.1, International Council of the Aeronautical Sciences, August 1982.

[2] Payne, B. W., "Designing a Load Alleviation System for a Modern Civil Aircraft,” ICAS paper 86-5.2.3, International Council of the Aeronautical Sciences, September 1986.

[3] Mack, M. D., Seetharam, H. D., Kuhn, W. G., and Bright, J. T., “Aerodynamics of Spoiler Control Devices,” AIAA paper 1979-1873, AIAA Aircraft Systems and Technology Meeting, August 1979. https://doi.org/10.2514/6.1979-1873

[4] Choi, S.-W., and Chang, K.-S., "Navier-Stokes Computation of a Rapidly Deploying Spoiler," Journal of Aircraft, Vol. 37, No. 4, 2000, pp. 655-661. https://doi.org/10.2514/2.2648

[5] Siddalingappa, S. R., and Hancock, G. J., “An Introduction to the Aerodynamics of Spoilers,” Paper QMC EP-1034, Department of Aeronautical Engineering, Queen Mary College, London, August 1980.

[6] Wentz, W., Jr., Ostowari, C., and Seetharam, H. C., "Effects of Design Variables on Spoiler Control Effectiveness, Hinge Moments and Wake Turbulence,” AIAA paper 1981-0072, 19 th AIAA Aerospace Sciences Meeting, January 1981. https://doi.org/10.2514/6.1981-72

[7] Hancock, G. J., "Dynamic Effects of Controls,” AGARD-R-711 paper 4, AGARD Special Course on Aerodynamic Characteristics of Controls, July 1983.

[8] Mabey, D. G., "Experimental Methods to Determine Control Effectiveness in Wind Tunnels," AGARD-R-711 paper 5, AGARD Special Course on Aerodynamic Characteristics of Controls, July 1983.

[9] McLachlan, B. G., Karamcheti, K., Ayoub, A., and Hadjidakis, G., "A Study of the Unsteady Flow Field of an Airfoil with Deflected Spoiler," AIAA paper 1983-2131, 10 ${ }^{\text {th }}$ AIAA Atmospheric Flight Mechanics Conference, August 1983. https://doi.org/10.2514/6.1983-2131

[10] Kalligas, K., “The Dynamic Characteristics of Two-Dimensional Spoilers at Low Speeds," Ph.d. thesis, Department of Aeronautical Engineering, University of Bristol, December 1986. 
[11] Lee, C. S., and Bodapati, S., "Experimental Investigations of the Flowfield of an Airfoil with Spoiler," AIAA Journal, Vol. 25, No. 4, 1987, pp. 1411-1416. https://doi.org/10.2514/3.9797.

[12] Consigny, H., Gravelle, A., and Molinaro, R., "Aerodynamic Characteristics of a Moving Two-Dimensional Spoiler in Subsonic and Transonic Flow," Journal of Aircraft, Vol. 21, No. 9, 1984, pp. 687-683. https://doi.org/10.2514/3.45015.

[13] Costes, M., Gravelle, A., Philippe, J. J., Vogel, S., and Triebstein, H., "Investigation of Unsteady Subsonic Spoiler and Flap Aerodynamics,” Journal of Aircraft, Vol. 24, No. 9, 1987, pp. 629-637. https://doi.org/10.2514/3.45488.

[14] Nelson, C. F., Koga, D. J., and Eaton, J. K., “Unsteady, Separated Flow Behind an Oscillating, Two-Dimensional Spoiler,” AIAA Journal, Vol. 28, No. 5, 1990, pp. 845-852. https://doi.org/10.2514/3.25128.

[15] Yeung, W. W. H., Xu, C., and Gu, W., "Reduction of Transient Adverse Spoiler Effects," Journal of Aircraft, Vol. 34, No. 4, 1997, pp. 479-484. https://doi.org/10.2514/2.2216

[16] Jordan Jr., F. L., Gato, W., Masiello, M. F., O’Rourke, M. J., and White, E. R., "Experimental Investigation of Unsteady Aerodynamics on a Flap Element Induced by Rapid Spoiler Deflection,” AIAA paper 1994-1886, 12 ${ }^{\text {th }}$ AIAA Applied Aerodynamics Conference, June 1994. https://doi.org/10.2514/6.1994-1886.

[17] Scott, R. C., Hoadley, S. T., Wieseman, C. D., and Durham, M. H., “The Benchmark Active Controls Technology Model Aerodynamic Data," AIAA paper 97-0829, $35^{\text {th }}$ AIAA Aerospace Sciences and Meeting Exhibit, January 1997. https: //doi.org/10.2514/6.1997-829

[18] NATO-RTO, "Verification and Validation Data for Computational Unsteady Aerodynamics," RTO Technical Report 26, Applied Vehicle Technology Panel (AVT) Task Group AVT-010, October 2000. https://doi.org/10.14339/RTO-TR-026

[19] Ok, H., and Eberhardt, D. S., "Calculation of the Flowfield Around an Airfoil with Spoiler," AIAA paper 93-0527, 31 ${ }^{s t}$ AIAA Aerospace Sciences Meeting, January 1993. https://doi.org/10.2514/6.1993-527

[20] Kim, J. H., and Rho, O. H., "Numerical Simulation of Flowfield Around Airfoil with Stationary or Oscillating Spoiler,” Journal of Aircraft, Vol. 35, No. 5, 1998, pp. 704-711. https://doi.org/10.2514/2.2380.

[21] Choi, S.-W., Chang, K.-S., and Ok, H., "Parametric Study of Transient Spoiler Aerodynamics with Two-Equation Turbulence Models," Journal of Aircraft, Vol. 38, No. 5, 2001, pp. 888-894. https://doi.org/10.2514/2.2848

[22] Chow, R., and van Dam, C. P., “Unsteady Computational Investigations of Deploying Load Control Microtabs,” Journal of Aircraft, Vol. 43, No. 5, 2006, pp. 1458-1469. https://doi.org/10.2514/1.22562

[23] Xu, C., and Yeung, W. W. H., "Unsteady Aerodynamic Characteristics of Airfoil with Moving Spoilers," Journal of Aircraft, Vol. 36, No. 3, 1999, pp. 530-540. https://doi.org/10.2514/2.2488

[24] Favre, F., Antepara, O., Lehmkuhl, O., Borrell, R., and Oliva, A., “On the Fast Transient Spoiler Deployment in a NACA0012 Profile Using LES Techniques Combined with AMR and IMB Methods," Proceedings of the $\sigma^{\text {th }}$ European Conference on Comutational Fluid Dynamics, ECFD VI, 2014. 
[25] Gand, F., "Zonal Detached Eddy Simulation of a Civil Aircraft with a Deflected Spoiler," AIAA Journal, Vol. 51, No. 3, 2013, pp. 697-706. https://doi.org/10.2514/1.J052106.

[26] Wilkinson, W. M., Lines, T. R., and Yu, N. J., “Navier-Stokes Calculations for Massively Separated Flows,” AIAA paper 96-2383, $14^{\text {th }}$ AIAA Applied Aerodynamics Conference, January 1996. https://doi.org/10.2514/6.1996-2383

[27] Bartels, R. E., and Schuster, D. M., "Comparison of Two Navier-Stokes Methods with Benchmark Active Control Technology Experiments," Journal of Guidance, Control and Dynamics, Vol. 23, No. 6, 2000, pp. 1094-1099. https://doi.org/10.2514/2.4660

[28] Hantrais-Gervois, J.-L., Lepage, A., Ternoy, F., Carraz, G., and Jeanfaivre, G., “Assessment of Numerical Tools to Predict Control Surface Effectiveness," AIAA paper 2010-4816, $28^{\text {th }}$ AIAA Applied Aerodynamics Conference, June 2010. https://doi.org/10.2514/6.2010-4816

[29] Tian, Y., Feng, P., Liu, P., Hu, T., and Qu, Q., "Spoiler Upward Deflection on Transonic Buffet Control of Supercritical Airfoil and Wing," Journal of Aircraft, Vol. 54, No. 3, 2017, pp. 1227-1231. https://doi.org/10.2514/1.C033574

[30] Geisbauer, S., and Loeser, T., “Towards the Investigation of Unsteady Spoiler Aerodynamics,” AIAA paper 2017-4229, 35 AIAA Applied Aerodynamics Conference, June 2017. https://doi.org/10.2514/6.2017-4229

[31] Gerhold, T., "Overview of the Hybrid RANS Code TAU," Notes on Numerical Fluid Mechanics and Multidisciplinary Design, Vol. 89, edited by N. Kroll and J. Fassbender, Springer, 2005, pp. 81-92. https://doi.org/10.1007/3-540-32382-1_5.

[32] Allmaras, S. R., Johnson, F. T., and Spalart, P. R., "Modifications and Clarifications for the Implementation of the SpalartAllmaras Turbulence Model," Proceedings of the $7^{\text {th }}$ International Conference on Computational Fluid Dynamics, ICCFD7, 2012.

[33] Menter, F., "Two-Equation Eddy-Viscosity Turbulence Models for Engineering Applications," AIAA Journal, Vol. 32, No. 8, 1994, pp. 1598-1605. https://doi.org/10.2514/3.12149

[34] Togiti, V., and Eisfeld, B., "Assessment of g-Equation Formulation for a Second-Moment Reynolds Stress Turbulence Model," AIAA paper 2015-2925, 22 ${ }^{\text {nd }}$ AIAA Computational Fluid Dynamics Conference, June 2015. https://doi.org/10.2514/6.20152925

[35] Cecora, R.-D., Radespiel, R., Eisfeld, B., and Probst, A., "Differential Reynolds-Stress Modeling for Aeronautics," AIAA Journal, Vol. 53, No. 3, 2015, pp. 739-755. https://doi.org/10.2514/1.J053250

[36] Spiering, F., "Development of a Fully Automatic Chimera Hole Cutting Procedure in the DLR TAU Code," New Results in Numerical and Experimental Fluid Mechanics X, Notes on Numerical Fluid Mechanics and Multidisciplinary Design, Vol. 132, 2016. https://doi.org/10.1007/978-3-319-27279-5_51

[37] Fillola, G., Carrier, G., and Dor, J., "Experimental Study and Numerical Simulation of Flow Around Wing Control Surface," ICAS paper 2006-3.6.2, International Council of the Aeronautical Sciences, September 2006. 